\title{
A review on synchronous CDMA systems: optimum overloaded codes, channel capacity, and power control
}

\author{
Seyed Amirhossein Hosseini", Omid Javidbakht, Pedram Pad and Farrokh Marvasti
}

\begin{abstract}
This paper is a tutorial review on important issues related to code-division multiple-access (CDMA) systems such as channel capacity, power control, and optimum codes; specifically, we consider optimum overloaded codes that achieve errorless transmission in the absence of noise for the binary and nonbinary cases. A survey of lower and upper bounds for the sum channel capacity of such systems is given in the presence and absence of channel noise. The asymptotic results for the channel capacity are also investigated. The channel capacity, errorless transmission codes, and power estimation for near-far effects are also explored. The emphasis of this tutorial review is on the overloaded CDMA systems.
\end{abstract}

Keywords: Code Division Multiple Access (CDMA), Optimum codes, channel capacity bounds, near-far effects, power control

\section{Introduction}

code-division multiple access (CDMA) has been the most important multiple access technology for the 3rd generation GSM and American Cellular systems [1]. Optical CDMA systems have become an alternative multiple access for fiber optics and optical wireless systems [2-4].

In CDMA systems, each user is assigned a unique code signature that consists of a number of chips. The signature length (also called chip rate) is defined as the number of chips in each signature code. Each user signature is multiplied by the respective data, and the transmitted vectors are added up in the common channel. The resultant vector is then observed at the received user end. In order to decode the received signal, the detector of the received user should know its own unique signature. These codes should be designed such that the cross-correlations between the code of the desired user and the codes of the other users are minimal.

For the wireless case, the most well-known binary (Endnote a) code for the synchronous case is Hadamard

\footnotetext{
* Correspondence: ahosseini@ee.sharif.edu

Advanced Communications Research Institute (ACRI) and Department of Electrical Engineering Sharif University of Technology, Tehran, Iran
}

orthogonal code that is appropriate for fully and underloaded CDMA systems. (Endnote b) But because of bandwidth limitation in the communication systems, we are interested in finding codes that can support more users than the chip rate (overloaded case). In the overloaded case, we cannot use Hadamard codes; Even random codes create interference that cannot be eliminated completely [5-7]. Optical orthogonal codes (OOC) [3,8] are not really orthogonal; however, using interference cancelation, we can remove interference completely. Most of the research in the overloaded case is related to code design and multi-access interference (MAI) cancelation in order to decrease the probability of error. Examples of these types of codes are pseudo random spreading $[9,10]$, Welsh Bound Equality (WBE) codes with minimal total square correlation (TSC) [11-14], OCDMA [15-17], Multiple-OCDMA [18], and PN/ OCDMA [19] signature sets. None of the above codes guarantee errorless transmission in the absence of channel noise for overloaded CDMA systems. There are also some codes that are not designed upon cross-correlation and are designed such that they can provide one-to-one transformation such as codes for overloaded wireless $(\mathrm{COW})$ and codes for overloaded optical (COO) codes [20-23]. By using these codes, we can achieve errorless 
transmission in the absence of channel noise for highly overloaded systems. The general case where both signature matrices and input vectors are complex is also discussed in $[20,21]$.

The decoding process of CDMA systems is more complex than the decoding of single user transmission systems. Since the transmitted signal is constructed upon a specific combination of all user data, multi user detection (MUD) is needed to decode the received vector. In MUD, the data of each user are extracted from the received vector at the receiver end, [24]. A popular method for MUD is maximum likelihood (ML) decoding where the received vector is compared with all possible input vectors and the most probable one is chosen as the transmitted vector.

There are many comprehensive theorems for capacity regions of multiple access channel (MAC), but the capacity region is not known in terms of specific model parameters. The capacity region includes a set of information rates such that simultaneous reliable communication is possible for each user. This problem was developed by Ahlswede [25,26] and Liao [27] on a twouser discrete memoryless channel. Cover $[28,29]$ and Wyner [30] obtained an explicit expression for the capacity region of the Gaussian discrete memoryless MAC. In [31], Verdu found the capacity of the CDMA channel as a function of cross-correlation between the assigned signature waveforms and their signal-to-noise ratios (SNRs) for the symbol synchronous case and for inputs with power constraints. The same author [32] found the capacity region for symbol asynchronous case for Gaussian distributed inputs with power constraints; in these two papers, Verdu showed that the achievable rates depend only on the correlation matrix of the spreading coefficients. He discussed about the complexity of MUD receivers in his book [5]. The analysis of spectral efficiency (defined as bits per chip that can be transmitted reliably) for linear detector is done in [33]. For finite number of users and real inputs and signatures, an upper bound for the sum capacity has been defined in [34]. The extension of the sum capacity bounds for asymmetric user power constraint is given in [35]. In [36], the authors have found lower and upper bounds for spectral efficiency (defined as the sum capacity by the authors) under quasi-static fading environments, channel estimation, and training sequences; the bound evaluations are based on the works of $[37,38]$. Another important issue is to obtain lower and upper bounds for the sum capacity in the asymptotic case, where the number of users $(n)$ and the number of chips $(m)$ go to infinity while $n / m$ are constant. Tanaka obtained a formula for the normalized sum capacity by applying the replica method from statistical physics to this problem [39]. Tanaka evaluated the performance of a class of
CDMA MUD in the large scale system limit analytically. These results were later extended in [24] to include unequal powers and fading channels. In $[40,41]$, progress was made toward a rigorous derivation of Tanaka's capacity formula. In [42], the authors have shown that, for large systems, the capacity concentrates around its mean, i.e., a random signature matrix results in a capacity very close to the "mean capacity" with high probability. The same authors in [41] proved that Tanaka's formula is an upper bound to the capacity for all values of the parameters and obtained various concentration theorems for the large scale system. In $[20,21]$, the authors have found lower and upper bounds for the channel capacity in the general case without using the statistical physics approach for both noiseless and noisy channels. In [43], the same task was performed for the binary case.

Another important issue in a communication system are near-far effects. In a cellular network, every user has a different distance from the base station, which makes the received power for each user to be different from each other. These phenomena are called the near-far effects. Most of the works on the near-far effects in CDMA are related to new methods for decoding such as MMSE in combination with successive interference cancelation (SIC) [44] and blind adaptive interference suppression [45]. In order to combat near-far effects, the method of isolation bit insertion was introduced in [46]. In [47], the authors have found lower and upper bounds for near-far distance of an MMSE detector. These methods depend on estimating the received power for each user. In [48], a new class of codes that are resistant to the near-far effects was presented. Another challenging problem is to derive the channel capacity of CDMA systems in the presence of near-far effects. The authors of [49] have presented lower and upper bounds for the channel capacity of overloaded CDMA systems with near-far effects. Some power control techniques are also used to combat these effects; however, because the techniques are imperfect, the receiver needs to estimate the received power for each user for a proper decoding. This problem becomes more critical in the overloaded case. The channel model that was used in $[48,49]$ is much worse than what happens in practice since it was proposed that the stochastic process of the power change for each user is a white process; the received value of the power for each user is independent of its value in any other time index. Nevertheless, in practice, the user powers vary slowly in comparison with the data rate. In [50], a new method has been presented in order to estimate the power of each user. The only information at the receiver is the signature matrix. The authors have assumed that the receiver knows the covariance matrix of noise. However, they 
have also showed that even without knowing the covariance matrix of noise, the power of each user can be calculated. But in this case, the maximum allowable number of users decreases. An iterative power estimating method was used in [50]. In each iteration, they extracted the estimated power obtained from the previous power estimation.

The organization of this tutorial review is as follows: In Section II, we take a look at generalized codes for overloaded wireless (GCO) which guarantee errorless transmission in an ideal channel and propose an ML decoding scheme for a special class of these codes. In this section, we will also survey the most popular case when the signature matrices and input vector entries are binary including active user detection. (Endnote c) In Section III, we will review the evaluation of the channel capacity of CDMA systems with and without channel noise. The asymptotic case will also be considered in this section. In Section IV, we will review a code that can support errorless transmission with the near-far effects. The channel capacity lower and upper bounds are also reviewed in the same section with near-far effects. Also in the same section, we will consider the received user power estimation for the highly over loaded CDMA systems. The summary, conclusion, and future works are in Section V.

\section{Introduction to optimum codes for CDMA transmission}

For combating the problem of bandwidth limitation in wireless and optical CDMA systems, we are interested to use optimum codes with a large overloading factor. In this section, we will review such signature codes for different cases. In the first part, the most general case is considered. In this case, the signature entries and input alphabets are completely arbitrary (real or complex) $[20,21]$. But in real communication systems, we deal with more practical applications, and therefore, some special cases such as the binary input and binary signature CDMA systems [22] and the codes being able to detect the active users in a CDMA system [23] are considered. We will also take a brief look at CDMA systems with WBE (Welsh Bound Equality) codes that are optimum for analog inputs [11]. At the end of this section, the ML detection algorithms for the signature codes are presented.

\section{A Generalized codes for overloaded wireless CDMA systems}

In this section, we will study a general class of optimum signature codes for CDMA transmission in an overloaded system. Optimality implies that such codes should provide errorless transmission in the absence of additive noise. In order to explain how these codes are optimum, a geometric interpretation of the transmission system is used. Assume that the number of users and chips are $n$ and $m$, respectively; for the overloaded case, $n>m$. The signature code is thus an $m \times n$ matrix, and the noiseless channel expression for this transmission is represented by $Y=\mathbf{C} X$ where $X$ is the $n \times 1$ user input vector, $Y$ is the $m \times 1$ received vector, and $\mathbf{C}$ is the $m \times$ $n$ signature matrix. Furthermore, assume that the inputs are from a given set $\mathcal{I}$ and the signature entries are taken from the given set $\mathcal{S}$. The $n$-dimensional user vector consisting of the user entries taken from the set $\mathcal{I}^{n}$ can be represented as vertices of a hypercube. This hypercube is mapped into an $m$-dimensional space since it is multiplied by the $m \times n$ signature matrix. In order for the transmission to be errorless, this mapping has to be one-to-one. Such matrices are called $\operatorname{GCO}(m, n, \mathcal{I}, \mathcal{S})$, and we will find $m$ and $n$ such that $\operatorname{GCO}(m, n, \mathcal{I}, \mathcal{S})$ codes exist. The following theorem shows a construction method for GCO matrices, which is proved in $[20,21]$.

By defining $\tilde{\mathbf{I}}_{m}$ to be the first $m$ - 1 columns of the $m$ $\times m$ identity matrix $\mathbf{I}_{m}, \tilde{\mathbf{J}}_{m}$ to be the first $m-1$ columns of the $m \times m$ all one matrix $\mathbf{J}_{m}, \tilde{\mathbf{K}}_{m}$ to be $2 \tilde{\mathbf{I}}_{m}-\tilde{\mathbf{J}}_{m}$ and $\tilde{\mathbf{0}}_{m}$ to be the first $m-1$ columns of the $m \times m$ zero matrix $\mathbf{0}_{m}$, the following theorem shows a general method for constructing GCO matrices

Theorem 1 For integer sets of $\mathcal{I}, \mathcal{S}$ assume that $\mathbf{C}$ is a $\operatorname{GCO}(m, n, \mathcal{I}, \mathcal{S})$ matrix, $\mathbf{H}_{w}$ is a $w \times w$ Hadamard matrix, and $k$ is an integer number. Also, suppose that $\pm k \mathcal{S} \subset \mathcal{S}^{\prime}$ and $d$ is the largest nonzero integer such that $\frac{\mathcal{I}-\mathcal{I}}{d} \subset \mathbb{Z}$. If $\mathbf{M}_{1}, \mathbf{M}_{2}, \ldots, \mathbf{M}_{w}$ are matrices with entries from $\mathcal{S}^{\prime}$ such that $\sum_{i=1}^{w} \mathbf{M}_{i}=t \mathbf{I}_{m}$ where $t$ is any integer, then the following matrix $\mathbf{B}$ is a $\mathrm{GCO}\left(w m, w n+m, \mathcal{I}, \mathcal{S}^{\prime}\right)$ matrix (Endnote d)

$$
\mathbf{B}=\left[\begin{array}{cc} 
& \mathbf{M}_{1} \\
& \mathbf{M}_{2} \\
k \mathbf{H}_{w} \otimes \mathbf{C} & \vdots \\
& \mathbf{M}_{w}
\end{array}\right],
$$

If

$$
\left\{\frac{u w k}{t}: u \in \mathbb{Z}\right\} \cap \frac{\mathcal{I}-\mathcal{I}}{d}=0 .
$$

Moreover, when $\mathcal{S}$ consists of only odd numbers and $\sum_{i=1}^{w} \mathbf{M}_{i}=t \tilde{\mathbf{I}}_{m}$, then $\mathbf{B}$ is a $\operatorname{GCO}\left(w m, w n+m, \mathcal{I}, \mathcal{S}^{\prime}\right)$ matrix if

$$
\left\{\frac{2 u w k}{t}: u \in \mathbb{Z}\right\} \cap \frac{\mathcal{I}-\mathcal{I}}{d}=0 .
$$

Since the creation of large GCO matrices is very complicated, in $[20,21]$, a method was introduced to create 
large sized GCO matrices from smaller ones. This method is presented below:

Theorem 2 If $\mathbf{A}$ is a $\operatorname{GCO}(m, n, \mathcal{I}, \mathcal{S})$ matrix and $\mathbf{P}$ is a $w \times w$ invertible matrix with entries belonging to the set $\mathcal{R}$, then $\mathbf{P} \otimes \mathbf{A}$ is a $\operatorname{GCO}(m w, n w, \mathcal{I}, \mathcal{S} \cdot \mathcal{R})$ matrix where $\mathcal{S}$. $\mathcal{R}$ is a set of all products of the elements of $\mathcal{S}$ and $\mathcal{R}$.

So far, the existence of such GCO codes has been shown, and matrices with specific overloading factors have been derived. But we can find signature matrices that can support a much larger number of users while the transmission is still errorless in the absence of noise. However, the number of users cannot exceed a maximum value because the vertices of the $n$-dimensional hypercube cannot be mapped to disjoint points. In $[20,21]$, the authors have shown an inequality that introduces an upper bound for the number of users for a GCO matrix with a fixed number of chips:

Theorem 3 If there exists a $\operatorname{GCO}(m, n, \mathcal{I}, \mathcal{S})$, then

$$
n \log _{2}|\mathcal{I}| \leq m \max _{\left(a_{1}, \ldots, a_{n}\right) \in \mathcal{S}^{n}} \mathbb{H}\left(Y_{1}\right),
$$

where $\mathbb{P}\left(Y_{1}=l\right)$ is equal to the number of solutions of equation $\sum_{j=1}^{n} a_{j} X_{j}=l$ in $\mathcal{I}$, divided by $|\mathcal{I}|^{n}$.

\section{B Codes for overloaded wireless and optical CDMA}

As mentioned earlier, practical communication systems predominantly use binary input and signature alphabets. We can apply the previous theorems to the binary case. In the wireless case, $\mathrm{COW}$ and in the optical case, $\mathrm{COO}$ CDMA systems are studied in [22]. In overloaded CDMA systems with binary inputs, we can use COW/ $\mathrm{COO}$ signature matrices. By using these binary matrices, we can achieve errorless transmission in the absence of noise.

It can be shown that there exists an $m \times n \mathrm{COO}$ matrix for the optical CDMA if there is an $m \times n$ COW matrix for wireless CDMA just by substituting all -1 entries of the COW matrix by 0 [22]. Thus, from now on, we will only discuss the COW matrices. Since the signature matrix $\mathbf{C}$ must be one-to-one, the necessary and sufficient condition is that $\operatorname{KerC} \cap\{-1,0,1\}^{n}=\{0\}^{n}$, where $\mathrm{Ker} \mathbf{C}$ is the null space of $\mathbf{C}$.

The authors of [22] have applied Theorem 2 in order to develop a large COW matrix from a smaller one. In this case, the matrix $\mathbf{C}$ is an $m \times n$ COW matrix and $\mathbf{T}$ is an invertible $k \times k$ matrix. Thus, $\mathbf{C} \otimes \mathbf{T}$ is a $k m \times k n$ COW matrix, where the overloading factor remains constant. However, the following theorem shows the existence of COW matrices with much higher overloading factor [51].

Theorem 4 Assume that $\mathbf{C}$ is an $m \times n$ COW matrix and $\mathbf{H}_{2}=\left[\begin{array}{ll}+1 & +1 \\ +1 & -1\end{array}\right]$ and $\mathbf{V}_{2} m \times(m-1)=\left[v_{i j}\right]$ such that

$$
v_{i j}= \begin{cases}-1 & j<i \leq m \\ +1 & \text { otherwise }\end{cases}
$$

Then, $\mathbf{D}=\left[\mathbf{H}_{2} \otimes \mathbf{C ~ V}\right]$ is an $2 m \times(2 n+m-1) \mathrm{COW}$ matrix.

By applying the above method on $\mathbf{C}_{4 \times 5} \mathrm{COW}$ matrix four times, a large overloaded signature matrix $\mathbf{C}_{64} \times 193$ is created.

In COW matrices, there exists also an upper bound for the overloading factor that is presented in the following inequality [22]:

$$
n<-m\left(\sum_{t=0}^{n} \frac{\left(\begin{array}{c}
n \\
i
\end{array}\right)}{2^{n}} \log _{2} \frac{\left(\begin{array}{c}
n \\
i
\end{array}\right)}{2^{n}}\right),
$$

where $\left(\begin{array}{c}n \\ i\end{array}\right)=\frac{n !}{i !(n-i) !}$.

Figure 1 shows the upper bound for users stated in the inequality. This figure shows that for a chip rate of 64 , the number of users should be less than 268 , while from Theorem 4, we derived a $64 \times 193$ COW matrix.

\section{Codes for analog input CDMA systems}

So far, we have studied overloaded CDMA systems with finite input alphabets. But there is also a different class of CDMA systems with analog inputs. It is obvious that special signature codes are necessary for such systems. These codes are called Welsh bound equality (WBE) codes and can be also used for binary inputs (BWBE). It is shown that the capacity in such systems is maximized when the input has Gaussian distribution and the users have equal power [52]. According to a criterion called total squared correlation (TSC) [12], WBE codes have

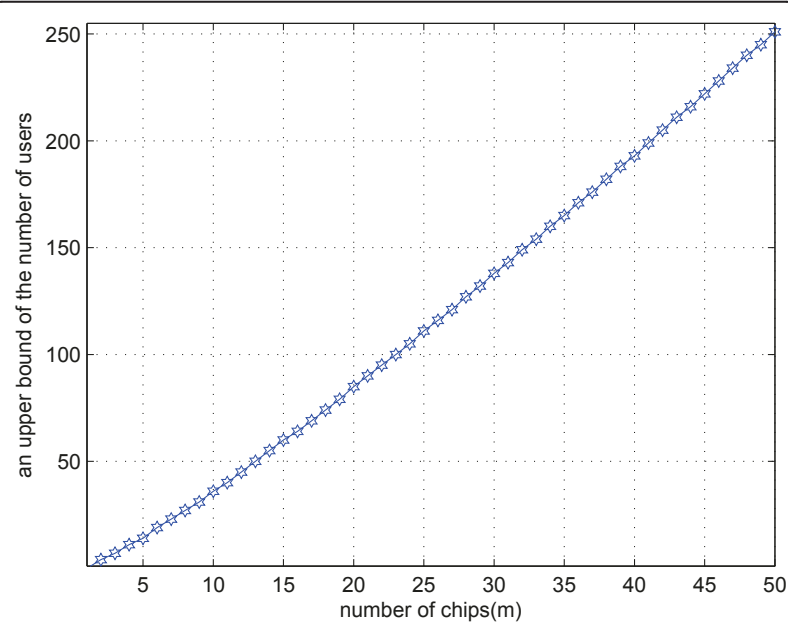

Figure 1 The upper bound for the number of users $n$ versus the spreading factor $m$. 
minimum correlation. Many methods have been introduced for constructing WBE codes either in the binary or nonbinary cases. In [11] it has been shown how to build large size Generalized WBE (GWBE) codes from smaller ones.

Two definitions of GWBE matrices are used in [11], namely a definition based on sum capacity and a definition based on TSC. Using these two definitions, we can derive another theorem, similar to Theorem 2 where large size GWBE codes can be constructed from small ones.

Theorem 5 If $\mathbf{S}$ is an $m_{1} \times n_{1}$ GWBE matrix $\left(n_{1}>m_{1}\right)$ for a CDMA system with user powers $p_{i}$ 's, and $\mathbf{T}$ is an $m_{2} \times n_{2}$ GWBE matrix $\left(n_{2}>m_{2}\right)$ with user powers $q_{j}^{\prime}$ s, then $\mathbf{S} \otimes \mathbf{T}$ is an $m_{1} m_{2} \times n_{1} n_{2}$ GBWE matrix for a CDMA system with user powers $p_{i} q_{j}$ 's for $1 \leq i \leq n_{1}$ and $1 \leq j \leq n_{2}$.

The theorem was proved for the overloaded case. This method results in a huge reduction in computational complexity since it requires much less computation than the direct construction of such matrices [53]. This algorithm can also be used for the binary input case for constructing BWBE codes.

\section{Overloaded CDMA with active user detection (COWDA)}

In many communication systems, such as ad-hoc networks [54], identification and localization of active nodes in the neighborhood of the transmitter is an important aspect. In [23], the authors have shown a new class of overloaded codes that not only detects the active users but also provides errorless transmission in a noiseless channel. These codes are called Codes for Overloaded Wireless CDMA with Detection of Active Users (COWDA).

For developing these kinds of codes, the authors of [23] have used the same geometric interpretation as in the COW/COO case. The only difference is that in this case the vertices of the hypercube are taken from the set $\{0, \pm 1\}^{n}$ where 0 refers to the nonactive users. Now, a method is presented for creating this kind of signature matrices. The necessary and sufficient condition for the of a COWDA matrix $\mathbf{C}$ is that $\operatorname{Ker} \mathbf{C} \cap\{ \pm 2, \pm 1,0\}^{n}=\{0\}$ ${ }^{n}$. Thus, to find such COWDA matrices, KerC should be compared with $5^{n}$ vectors. But in [23], a method was shown in which the number of comparisons is reduced to $\frac{5^{(n-m)}-1}{2}$ if $\mathbf{C}=[\mathbf{A} \mathbf{B}]$, where $\mathbf{A}$ is an $m \times m$ invertible matrix and $\mathbf{B}$ is an $m \times(n-m)$ matrix. The following theorem presents a method to construct large size COWDA matrices from smaller ones:

Theorem 6 Assume that $\mathbf{C}$ is an $m \times n$ COWDA matrix and $\mathbf{H}_{4}=\mathbf{H}_{2} \otimes \mathbf{H}_{2}$ and $\mathbf{V}_{4 m \times(m-1)}=\left[v_{i j}\right]$ such that

$$
v_{i j}= \begin{cases}-1 & j<i \leq m \\ +1 & \text { otherwise }\end{cases}
$$

Then,, $\mathbf{D}=\left[\mathbf{H}_{4} \otimes \mathbf{C ~ V}\right]$ is an $4 m \times(4 n+m-1)$ COWDA matrix

Theorem 2 is also valid for the COWDA case and can be expressed as follows: If $\mathbf{C}$ is an $m \times n$ COWDA matrix and $\mathbf{T}$ is an invertible $k \times k$ matrix, then $\mathbf{C} \otimes \mathbf{T}$ is a $k m \times k n$ COWDA matrix.

By starting with the COWDA matrix $\mathbf{C}_{16 \times 22}$ and applying Theorem 6 , a $63 \times 103$ COWDA matrix can be obtained. In [51] the authors have conjectured that the number of users can increase up to 193.

Similar to the COW/COO case, there is an upper bound for the number of users for a COWDA matrix while the number of chips is constant in order for the transmission to be errorless in an ideal channel. Naturally, the maximum number of users in this case is less than the previous case because the number of input alphabets is more than the COW case, while the alphabets in the $m$-dimensional space remains the same. The upper bound is found to be [23]:

$$
n \leq-m\left(\sum_{k=-n}^{n} \frac{f(n, k)}{3^{n}} \log _{3} \frac{f(n, k)}{3^{n}}\right)
$$

where

$$
f(n, k)=\sum_{r=0}^{\left\lfloor\frac{n-k}{2}\right\rfloor}\left(\begin{array}{l}
n \\
r
\end{array}\right)\left(\begin{array}{l}
n-r \\
r+k
\end{array}\right)
$$

The below matrix shows a $4 \times 6$ COWDA matrix, where "+" denotes +1 and "-" denotes -1 .

$$
\left[\begin{array}{l}
++++++ \\
+--+0- \\
0+-+-+ \\
+0+---
\end{array}\right]
$$

\section{E ML decoding for finite input CDMA}

In order to decode the received vector in CDMA systems optimally, ML decoding is used. The received vector can be obtained from the following channel model,

$$
Y=\mathrm{C} X+N,
$$

where $Y$ is the $m \times 1$ received vector, $\mathbf{C}$ is the $m \times n$ signature matrix, $X$ is the $n \times 1$ input vector, and $N$ is the $m \times 1$ noise vector with Gaussian distribution with zero mean and auto covariance matrix $\sigma^{2} \mathbf{I}_{m}$. At the receiver, we should search among all possible inputs and find the one which minimizes $\|Y-\mathbf{C} \hat{X}\|^{2}$. As a result, we need to perform $2^{n}$ Euclidean distance measurements (for the binary input case) while this number will increase to $|\mathcal{I}|^{n}$ for the general case. This decoding 
method requires a high amount of computations that makes the receiver system very complex.

In order to reduce the complexity of decoding systems, Tensor Decoding Algorithm was used in [22,23]. This algorithm consists of two main steps that are described below:

Step 1: Suppose $\mathbf{D}_{k m \times k n}=\mathbf{P}_{k \times k} \otimes \mathbf{C}_{m \times n}$ where $\mathbf{P}$ is an invertible matrix and $\mathbf{C}$ is a $\mathrm{COW}$ matrix. Assume the channel model is $Y=\mathbf{D} X+N$, by multiplying both sides of the equation by, $\sqrt{k}\left(\mathbf{P}^{-1} \otimes \mathbf{I}_{m}\right)$ we obtain,

$$
Y^{\prime}=\sqrt{k}\left(\mathbf{P}^{-1} \otimes \mathbf{I}_{m}\right) Y=\sqrt{k}\left(\mathbf{I}_{k} \otimes \mathbf{C}\right) X+N^{\prime},
$$

where $N^{\prime}=\sqrt{k}\left(\mathbf{P}^{-1} \otimes \mathbf{I}_{m}\right) N$. This implies that the first $m$ entries of $Y^{\prime}$ depend on the first $n$ entries of $X$ and the first $m$ entries of $N$; the second $m$ entries of $Y^{\text {, }}$ depend on the second $n$ entries of $X$ and the second $m$ entries of $N$ and so on. Consequently, the algorithm was simplified by breaking it down to $p k$ smaller decoding problems. If the matrix $\mathbf{P}$ is Hadamard, the matrix $\sqrt{k}\left(\mathbf{P}^{-1} \otimes \mathbf{I}_{m}\right)$ will be unitary. As a result, the vector $N^{\prime}$ will have identical properties to $N$. Therefore, the $\mathrm{ML}$ decoding of $Y^{\prime}$ will be equivalent to the ML decoding of $Y$.

Step 2: The complexity of the decoding algorithm can be decreased further if the COW matrix can be written as $\mathbf{C}=[\mathbf{A} \mathbf{B}]$, where $\mathbf{A}$ is an $m \times m$ invertible matrix and $\mathbf{B}$ is an $m \times(n-m)$ matrix. Thus, $Y=\mathbf{C} X=\mathbf{A} X_{1}+$ $\mathbf{B} X_{2}$, where $X_{1}$ and $X_{2}$ are $m \times 1$ and $(n-m) \times 1$ vectors, respectively. It is obvious that $X_{1}$ can be written as $\mathbf{A}^{-1} \mathbf{Y}-\mathbf{A}^{-1} \mathbf{B} X_{2}$. In a noisy channel, we search for $X_{2}$ such that $\left\|\left(\mathbf{A}^{-1} Y-\mathbf{A}^{-1} \mathbf{B} X_{2}\right) \operatorname{sign}\left(\mathbf{A}^{-1} Y-\mathbf{A}^{-1} \mathbf{B} X_{2}\right)\right\|$ is minimized. Next, we can find $X_{1}$ by the equation $X_{1}=$ $\operatorname{sign}\left(\mathbf{A}^{-1} Y-\mathbf{A}^{-1} \mathbf{B} X_{2}\right)$. Thus, by applying this method, we decrease the number of Euclidean distance measurements from $2^{n}$ to $2^{n-m}$.

This decoding algorithm can be applied to all signature matrices explained in the previous sections. For active user detection, $X_{1}$ is obtained from the following equations: For the $i$ th user, we have

$$
\text { 1. If } i \leq m
$$

$$
\begin{cases}\left(\hat{X}_{1}\right)_{j}=\operatorname{sign}\left(\left(\mathbf{A}^{-1} Y-\mathbf{A}^{-1} \mathbf{B} \hat{X}_{2}\right)_{j}\right) & j=i \\ \left(\hat{X}_{1}\right)_{j}=\operatorname{softlim}\left(\left(\mathbf{A}^{-1} Y-\mathbf{A}^{-1} \mathbf{B} \hat{X}_{2}\right)_{j}\right) & j \neq i\end{cases}
$$

In this case $\hat{X}_{2}$ takes all vectors in $\{-1,0,+1\}^{k-l}$. 2. If $i>m$

$$
\left(\hat{X}_{1}\right)_{i}=\operatorname{softlim}\left(\left(\mathbf{A}^{-1} Y-\mathbf{A}^{-1} \mathbf{B} \hat{X}_{2}\right)_{i}\right)
$$

In the above equations, all entries of $\hat{X}_{2}$ belong to the set $\{-1,0,+1\}$ except for the $(i-m)$ th entry which is \pm 1 . In this algorithm, softlimgf is a soft limiter described as follows:

$$
\operatorname{softlim}(x)= \begin{cases}-1 & x<-\frac{1}{2} \\ 0 & -\frac{1}{2} \leq x \leq+\frac{1}{2} \\ +1 & +\frac{1}{2}<x\end{cases}
$$

Assume a COW matrix of size $(64,104)$ with the signature matrix $\mathbf{D}=\mathbf{H}_{8} \otimes \mathbf{C}_{8} \times 13\left(\mathbf{H}_{8}\right.$ is an $8 \times 8$ Hadamard matrix and $\mathbf{C}$ is a COW matrix). By using the direct ML decoding, we need to calculate $2^{104}$ Euclidean distance measurements, but by applying the Tensor Decoding Algorithm, this number reduces to $8 \times 2^{5}=$ $2^{8}$.

The detection algorithm for COWDA codes used in [23] was based on the assumption that in each transmission, the activeness of a user is independent of the previous transmission. This is a nonrealistic assumption since whenever a user is active, it remains active for a period of time and vice versa. Thus, the activeness of users are correlated in time. The above mentioned decoding algorithm is therefore appropriate for a case much worse than what happens in reality. In [55] a more realistic approach was used for decoding. $L$ subsequent transmissions were taken into account simultaneously and a Markov chain was created for these observations. If we assume the input vectors of $L$ subsequent transmissions to be $\underline{X}_{n \times L}=\left[X_{1}, X_{2}, \ldots, X_{\mathrm{L}}\right]$ and $\underline{Y}$ to be the matrix of the corresponding received vectors, then the decoder will be

$$
\underline{\hat{X}}=\underset{\underline{\tilde{X}}}{\operatorname{argmax}} \bar{f}(\underline{\tilde{X}} \mid \underline{Y}),
$$

where $\underline{\tilde{X}}$ belongs to the set $\{0, \pm 1\}^{n \times L}$ and $\bar{f}$ is the $n \times$ $L$ dimensional PDF of $Y$. According to Bayes rule and the fact that the channel is memoryless, we get

$$
\begin{aligned}
& \underline{\hat{X}}=\underset{\tilde{\underline{X}}}{\operatorname{argmax}} \mathbb{P}\left(\tilde{X}_{1}\right) f\left(Y_{1} \mid \tilde{X}_{1}\right) \\
& \times \prod_{i=1}^{L-1} \mathbb{P}\left(\tilde{X}_{i+1} \mid \tilde{X}_{i}\right) f\left(Y_{i+1} \mid \tilde{X}_{i}\right)
\end{aligned}
$$

This algorithm requires $3^{n L}$ computations which makes it very complex. But in [55], the Viterbi algorithm was used to decrease the complexity. In each step of the algorithm, we have $3^{n}$ states. The transition weight from step $i$ to step $i+1$ is $\mathbb{P}\left(\tilde{X}_{i+1} \mid \tilde{X}_{i}\right) f\left(Y_{i+1} \mid \tilde{X}_{i}\right)$. Thus, $3^{n}$ computations are required to find the path with the maximum weight and this task is performed for $L$ vectors. As a result, just $3^{n} 3^{n} L$ computations are required. It should be noted that $\mathbb{P}\left(\tilde{X}_{i+1} \mid \tilde{X}_{i}\right)$ just depends on the activeness or inactiveness of the user and therefore creates 2 instead of 3 states. Hence, the complexity reduces further to $2^{n} 3^{n} L$ computations. In addition, if the signature matrix satisfies the constraints of the first step of the Tensor Decoding Algorithm, the decoding problem 
can be broken down to several simpler decodings and thus, we need to perform $2^{n} 2^{m} 3^{n-m}$ computations. The same authors have also introduced three types of suboptimum decoders in order to reduce the amount of delay and memory needed for the decoding system. A low complexity decoding algorithm was also proposed in [56] for another class of signature codes. In the signature matrix introduced in [56], the signature vectors of each user have a hierarchical tree structure where each vector is correlated only with its direct ancestor and descendants, and the number of vectors at the bottom level is equal to the chip rate. It was also shown that if there are equal children emanating from each node in this tree structure, the number of levels of the tree is logarithmic in the number of users and the complexity is bounded by a very low order polynomial in $n$.

In order to compare the performance of the described signature codes, this part is dedicated to the comparison of the bit-error rate (BER) of systems that use random codes, BWBE codes, Hadamard codes, and COW codes. Figure 2 shows the BER versus $E_{b} / N_{0}$ for a $64 \times 72$ COW matrix. Also, other codes such as random codes, BWBE codes and Hadamard codes are shown on the same figure for comparison. Figure 3 is similar to Figure 2 except for a $64 \times 104$ signature code. In these figures, an iterative decoder for random and BWBE codes was used, while the Tensor Decoding Algorithm was applied for the COW case. The use of ML decoders for the $B W B E$ and random codes is not practical. As we see in these figures, BWBE codes perform slightly better than COW codes for $E_{b} / N_{0}$ values less than $10 \mathrm{~dB}$. However, for higher $E_{b} / N_{0}$ values, the performance of COW codes becomes better than BWBE codes. Since the mapping of BWBE codes, unlike the COW codes, is not invertible, for high values of $E_{b} / N_{0}$ (where the behavior of the systems tends to the noiseless case), the BER is saturated to a fixed value. These figures show the fact that for a

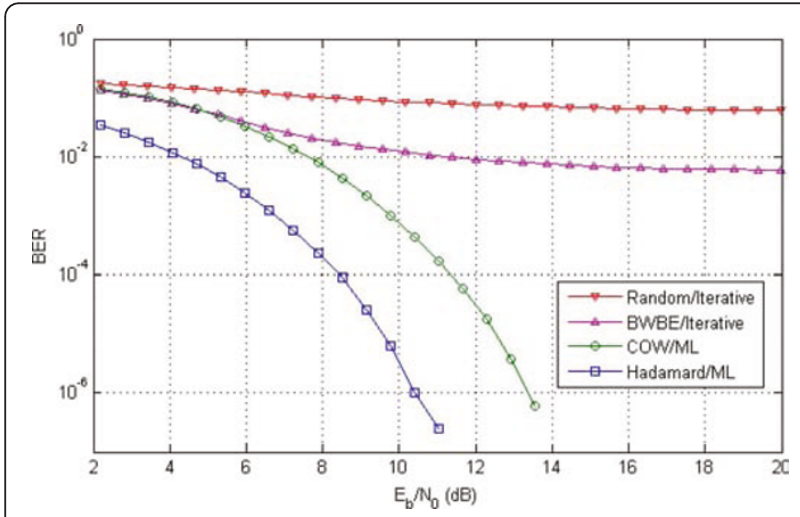

Figure 2 Bit-error rate versus $E_{b} / N_{0}$ for three classes of codes with 64 chips and 72 users (for comparison, Hadamard codes of size $64 \times 64$ is also simulated).

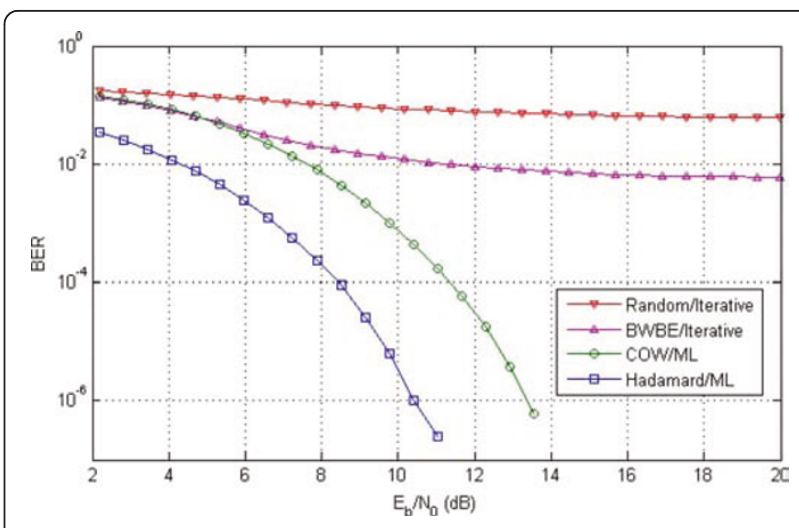

Figure 3 Bit-error rate versus $E_{b} / N_{0}$ for three classes of codes with 64 chips and 72 users (for comparison, Hadamard codes of size $64 \times 64$ is also simulated).

high overloading factor, the COW codes with their simple ML decoding outperforms codes with iterative decoding. BWBE codes perform better than random codes because of its minimum correlation property. For an overloading factor of $63 \%$, the Hadamard codes are 3 $\mathrm{dB}$ better than COW codes.

\section{Capacity bounds for CDMA systems}

In this section, we will review lower and upper bounds for the channel capacity of CDMA systems. In the first part, we define the concept of sum capacity. In the second part, lower and upper bounds are surveyed for binary and nonbinary CDMA transmission for the noiseless case and in the third part, the extension to the channel additive noise is discussed.

\section{A Definition of sum capacity}

In Multi Access Channels (MAC), additive noise and multi user interference are the main factors that cause disturbance in CDMA transmission. These factors affect the capacity of such channels. In [28], the authors have defined capacity regions to find all achievable transmission rates in such channels. In order to assign a single value as a measure of channel capacity, the sum capacity would be the best choice. The sum capacity is defined as the maximum sum of all user rates that can be achieved and is equal to $\max _{p_{1} \times p_{2} \times \cdots \times p_{n}} I\left(X_{1}, X_{2}, \ldots, X_{n} ; Y\right)$ where $p_{i}$ is the input distribution function of the $i$ th user.

The sum capacity for CDMA as a special case of MAC is also defined in [43]. For the noiseless case, the channel capacity of a system with binary signature matrix A will be equal to $C(m, n)=\mathbf{A} \in \mathcal{M}_{m \times n}( \pm 1) \max C(\mathbf{A})$, where $C(\mathbf{A})$ is the sum capacity of the channel. For the noisy case, we again use the channel model $Y=\frac{1}{\sqrt{m}} \mathbf{A} X+N$. The total power of the users is $\operatorname{tr}\left(\mathbb{E}\left(\frac{1}{m} \mathrm{~A} X X^{*} \mathbf{A}^{*}\right)\right.$ and the noise 
power is also modeled as $\mathbb{E}\left(N^{*} N\right)$. Thus, the multi user SNR at the receiver is defined in $[20,21]$ as

$$
\operatorname{SNR}=\frac{\operatorname{tr}\left(\mathbb{E}\left(\frac{1}{m} \mathrm{AXX} X^{*} \mathbf{A}^{*}\right)\right.}{\mathbb{E}(N * N)}
$$

where the entries of $N$ are i.i.d. random variables, $f(\cdot)$ is their common probability distribution function (pdf) with variance $\sigma_{f}^{2}$. This implies that the overall power at the receiver is equal to $m \sigma_{f}^{2}$. If $\frac{m}{n} \mathrm{SNR} \leq \eta$, we will have

$$
\operatorname{tr}\left(\mathbb{E}\left(\frac{1}{m} \mathbf{A X X} X^{*} \mathbf{A}^{*}\right) \leq n \eta \sigma_{f}^{2} .\right.
$$

For a given signature matrix $\mathbf{A}$ and $\eta$, the sum channel capacity will be defined as

$$
C(\mathbf{A}, \eta)=\max \left\{\left(\left\{(X ; Y) \mid X \sim p_{1}\left(x_{1}\right) \times p_{2}\left(x_{2}\right) \times \cdots \times p_{n}\left(x_{n}\right)\right\},\right.\right.
$$

such that the above inequality is satisfied.

The authors of [43] have tried to find the lower bounds for both noisy and noiseless channels in binary CDMA systems by choosing a random signature matrix and then derive the expected value of the sum capacity of the channel corresponding to this random matrix. In other words, the lower bound is the average sum channel capacity of a typical signature matrix. According to [41-43], the capacity of a channel with random signature matrix will be higher than the expected value with high probability.

All upper bounds that are derived for noisy and noiseless channels are based upon a conjecture which implies that the input vectors have uniform distribution [43]. In [41], the authors used this conjecture for the special case when the noise has Gaussian distribution. But for the general case, the authors of [43] considered this conjecture to be true for all noise distributions. Although this conjecture looks very simple, it is still an open problem.

\section{B Noiseless channel capacity bounds}

In this subsection, we will take a look at the lower and upper bounds for the sum capacity of general CDMA systems. These bounds are investigated further for several special cases such as COW matrices and active user detection systems. In the noiseless case, multi user interference is the only disturbance that has to be taken into account for CDMA transmission.

1 Lower bounds for the sum capacity of CDMA systems for the noiseless case

In the general mode where the signature alphabets and input vectors are not binary, the authors of $[20,21]$ first defined $p$ and $\pi$ as follows: Suppose that $\tilde{\mathcal{I}}$ is the difference set of $\mathcal{I}$ and is defined as:

$$
\tilde{\mathcal{I}}=\mathcal{I}-\mathcal{I}=\left\{i-i^{\prime} \mid i, i^{\prime} \in \mathcal{I}\right\}
$$

$\tilde{p}(\cdot)$ is defined as a probability on $\tilde{\mathcal{I}}$ to be the pdf of the difference of two independent random variables from the set $\mathcal{I}$ (the pdf of the random variables from $\mathcal{I}$ has the same distribution $p(\cdot))$. $\pi(\cdot)$ is a probability distribution on $\mathcal{S}$. The probability measure on the signature matrix $\mathcal{M}_{m \times n}$ is induced by choosing entries of the random matrix independently and with the same distribution $\pi(\cdot)$.

In $[20,21]$, a lower bound for the channel capacity for the general case was introduced which is stated in the following theorem:

\section{Theorem 7}

$$
C(m, n, \mathcal{I}, \mathcal{S}) \geq \sup _{p, \pi}\left\{-\log \mathbb{E}_{\tilde{X}}\left(\mathbb{P}\left(a^{T} \tilde{X}=0\right)^{m}\right)\right\},
$$

where $a \in \mathcal{S}^{n}$ and $\tilde{X} \in \tilde{\mathcal{I}}^{n}$ with i.i.d entries with distributions $\pi(\cdot)$ and $\tilde{p}(\cdot)$, respectively.

For the special case, where the input and signature matrix alphabets are finite, a simpler form for the above expression is derived in $[20,21]$.

For example, in the COW mode, the above lower bound simplifies to the lower bound obtained in [43]

$$
C(m, n) \geq n-\log _{2} \sum_{j=0}^{\left\lfloor\frac{n}{2}\right\rfloor}\left(\begin{array}{l}
n \\
2 j
\end{array}\right)\left[\frac{\left(\begin{array}{c}
2 j \\
j
\end{array}\right)}{2^{2 j}}\right]^{m}
$$

Another example could be the case where the input vectors are binary and $\mathcal{S}=\{0, \pm 1, \ldots, \pm p\}$. The lower bound has been derived in $[21,51]$.

\section{Conjectured upper bounds for the sum capacity of CDMA} systems for the noiseless case

In $[20,21]$, the authors introduced a theorem that presents a conjectured upper bound for the channel capacity in general

Theorem 8 In the absence of additive noise, if $\mathcal{I}=\left\{i_{1}, \ldots, i_{q}\right\}$ with distribution $p\left(i_{i}\right)=p_{i}$ and $\mathcal{S}=\left\{s_{1}, \ldots, s_{l}\right\}$, the upper bound is as follows:

$$
\begin{aligned}
& C(m, n, \mathcal{I}, \mathcal{S}) \leq \max \min (n \nVdash(\mathcal{I}), m \amalg(\tilde{f})) \\
& \sum_{i=1}^{l} u_{i}=n \\
& p(\cdot)
\end{aligned}
$$

in which

$$
\begin{aligned}
& \tilde{f}(z)=\sum_{\substack{q \\
\sum_{j=1}^{q} v_{i j}=u_{i} \\
1 \leq i \leq l}}\left(\prod_{k=1}^{l}\left(\begin{array}{c}
u_{k} \\
v_{k 1}, \ldots, v_{k q}
\end{array}\right)\right) \\
& \left(\prod_{k=1}^{q} p_{k}^{l} v_{k=1}\right) \delta\left(z-\frac{1}{\sqrt{m}}\left(\sum_{k=1}^{l} s_{k} \sum_{\alpha=1}^{q} v_{k \alpha} i_{\alpha}\right)\right),
\end{aligned}
$$


where $\delta$ is the Dirac function and $\amalg(f)$ is the entropy of the distribution $f$.

Also, when $s_{i}=\mathrm{e}^{\frac{2 \pi i}{l} \sqrt{-1}}$ and $l$ divides $n$, we conjecture that $u_{1}=u_{2}=\cdots=u_{l}=\frac{n}{l}$.

The above upper bound is simplified for COW matrices in [43]. For COW matrices, a simpler upper bound is obtained in [22]:

$$
C(m, n) \leq m\left(\frac{1}{2} \log _{2} n+\log _{2} \lambda\right)+1,
$$

where $\lambda$ is the unique positive solution of the equation

$$
(\lambda \sqrt{n})^{m}=m \mathrm{e}^{\frac{-\lambda^{2}}{2}} 2^{n+1} .
$$

Another example could be the case where the input vectors are binary and $\mathcal{S}=\{0, \pm 1, \ldots, \pm p\}$. The upper bound has been derived in $[21,51]$.

Although Equation (25) shows a tight upper bound on the channel capacity, in some regions, there are bounds that are a bit tighter than the above bound. These bounds are conceptually obvious and are shown below:

$$
\begin{aligned}
& C(m, n) \leq n \\
& C(m, n) \leq m \log _{2} n+1
\end{aligned}
$$

The conjectured upper bound that was introduced in Equation (25) was a special case when the noise variance is zero without restricting the signature to have unity magnitude. Another conjectured upper bound that is more general and does not depend on the symbol alphabet is introduced in $[20,21]$.

In Figure 4, The lower and upper bounds for a COW matrix with fixed chip rate $(m=64)$ are plotted versus the number of users. An interesting result that can be drawn from this figure is that the channel capacity increases almost linearly with the number of users until $n$ reaches a certain threshold value $n$ th. In this region, the errorless transmission is achieved and this implies that overloaded signature matrices do exist for these values of $m$ and $n$. As $n$ goes beyond this threshold value, the lower and upper bounds tend to diverge from each other. This implies that errorless transmission cannot be achieved anymore and multi user interference causes transmission errors. The lower bound for an $8 \times$ 13 COW matrix equals 12:164 bits. This shows the extreme tightness of this bound.

In Figure 5, the capacity bounds are sketched versus the number of chips for a fixed value of $n(n=220)$. In this figure, we see that as the number of chips increases and before it reaches a certain value $m$ th, the channel is lossy and errorless transmission is not achievable. This is due to the fact that when the number of chips is less than a certain value, the transform of the input vectors into an $m$ dimensional space is not one-to-one. But as $m$ increases over this threshold value, errorless transmission can be achieved. In this figure, we can also observe that in some regions, the upper bound introduced in (27) and (28) tends to be slightly tighter than the bound introduced in (25).

Figures 6 and 7 show the same facts as Figures 4 and 5 , respectively, but for several values of $m$ and $n$.

Figure 8 shows the normalized channel capacity bounds for binary CDMA systems. From this figure we can derive that systems with higher spreading factor can support more users.

In CDMA systems with relatively small values for $n$ and $m$ (small sale system) the sum channel capacity depends on the input and signature alphabets. In Figure 9 and 10, this dependence is shown for different systems with $m$ being equal to 32. In Figure 9, binary signature CDMA systems are considered while in Figure 10 the systems have binary inputs and ternary signatures.

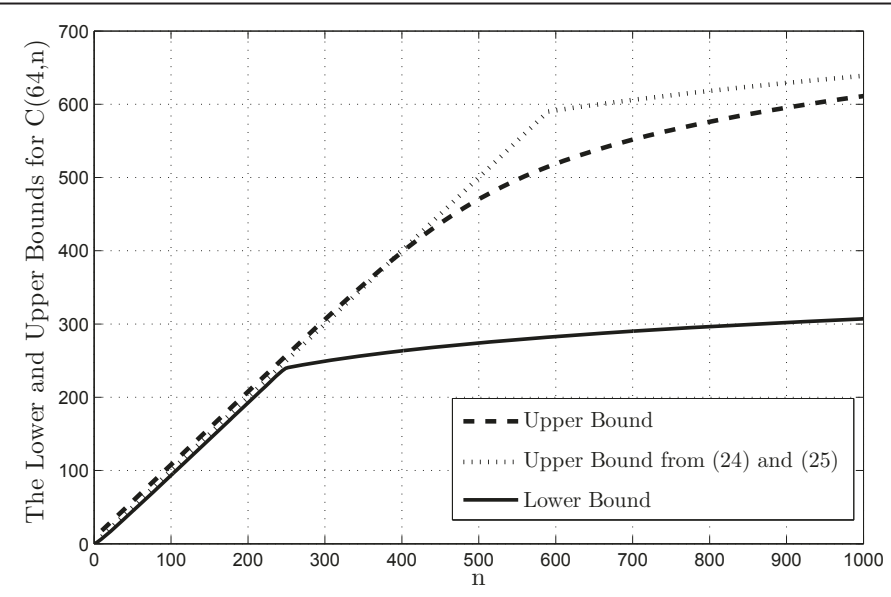

Figure 4 Lower and upper bounds for the sum channel capacity versus the number of users $n$ for $m=64$ 


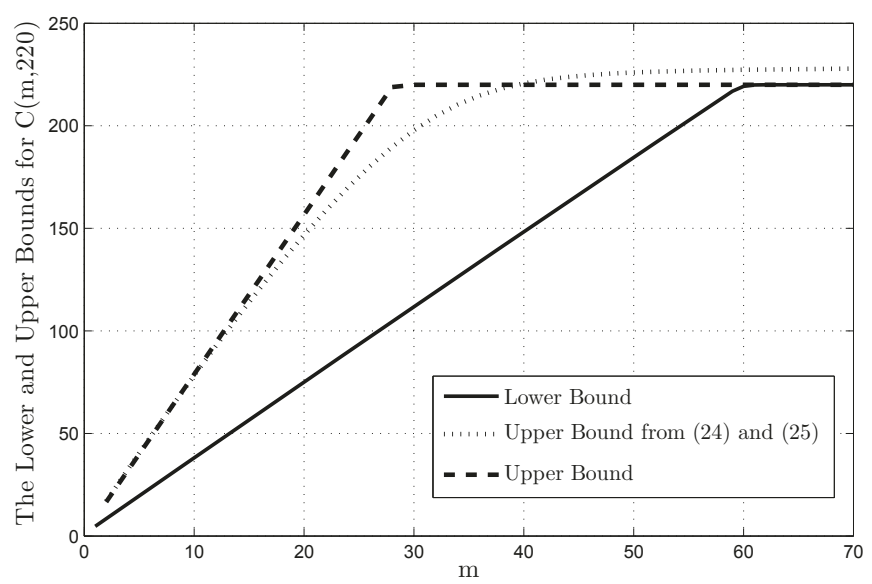

Figure $\mathbf{5}$ Lower and upper bounds for the sum channel capacity versus the chip rate $m$ for $n=220$.

\section{Noisy channel capacity bounds}

In the presence of noise, not only multi user interference but also additive noise can reduce the sum capacity of the system. In this subsection, lower and upper bounds sum channel capacity bounds with any arbitrary noise distribution are surveyed. However, only the Gaussian noise distribution is discussed in detail.

In the presence of additive noise, the calculation of channel capacity is a challenging problem. In [20,21], a lower and a conjectured upper bound for the general case is introduced and will be discussed later. Assume A $=r \mathbf{B}$ where $r$ is a fixed number and $\mathbf{B}$ is randomly chosen with distribution $\mathcal{P}^{\pi}$.

After taking expectation over $\mathcal{P}^{\pi}$, we have:

$$
r \leq \sqrt{\frac{\eta \sigma_{f}^{2}}{\left(\sigma_{p}^{2}+n \mu_{p}^{2}\right)}\left(\sigma_{\pi}^{2}+n \mu_{\pi}^{2}\right)},
$$

where $\mu_{p}$ and $\sigma_{p}$ are the mean and variance of the input distribution $p(\cdot)$, respectively, and $\mu_{\pi}$ and $\sigma_{\pi}$ are the mean and variance of the signature code distribution $\pi(\cdot)$.

1 Lower bounds for the sum capacity of CDMA systems for the noisy case

The authors of $[20,21]$ presented a theorem to obtain a lower bound for the most general case for any given input and signature matrix symbols and additive noise with arbitrary distribution:

\section{Theorem 9}

$$
\begin{aligned}
C(m, n, \mathcal{I}, \mathcal{S}, \eta) \geq \sup _{\pi, p} \sup _{q}\left[-m \mathbb{E}\left(q\left(N_{1}\right)\right)\right. \\
\left.\quad-\log \mathbb{E}_{\tilde{X}}\left(\left(\mathbb{E}_{b, N_{1}}\left(2^{-q\left(N_{1}-\frac{b^{T} \tilde{X}}{\sqrt{m}}\right)}\right)\right)^{m}\right)\right]
\end{aligned}
$$

Here, $q(\cdot)$ is any arbitrary function, $N_{1}$ is the first entry of the noise vector, $b$ and $\tilde{X}$ are vectors of length $n$ with i.i.d. entries of distribution $\pi(0)$ and $p(0)$.

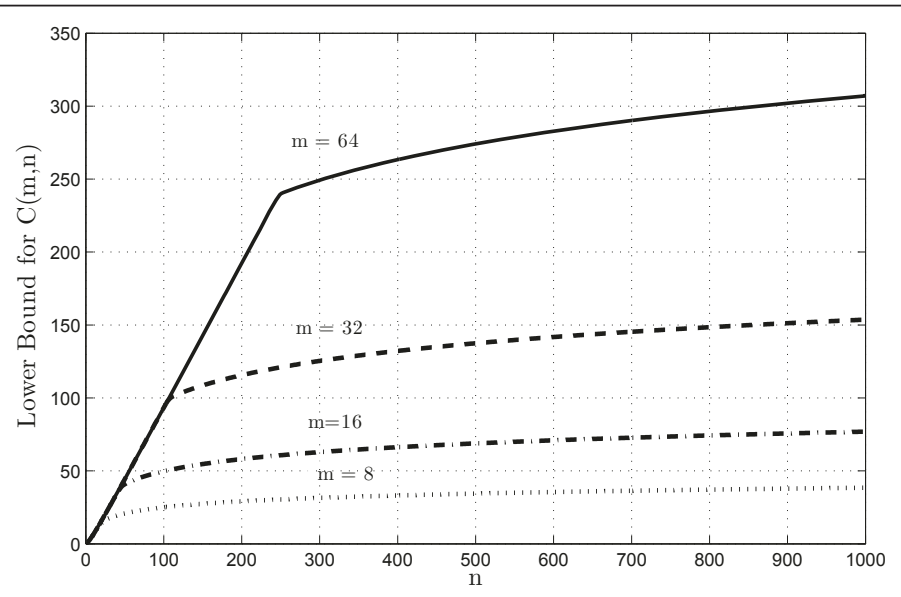

Figure 6 Plots of channel capacity lower bounds versus number of users $n$ for a various chip rates $m$. 


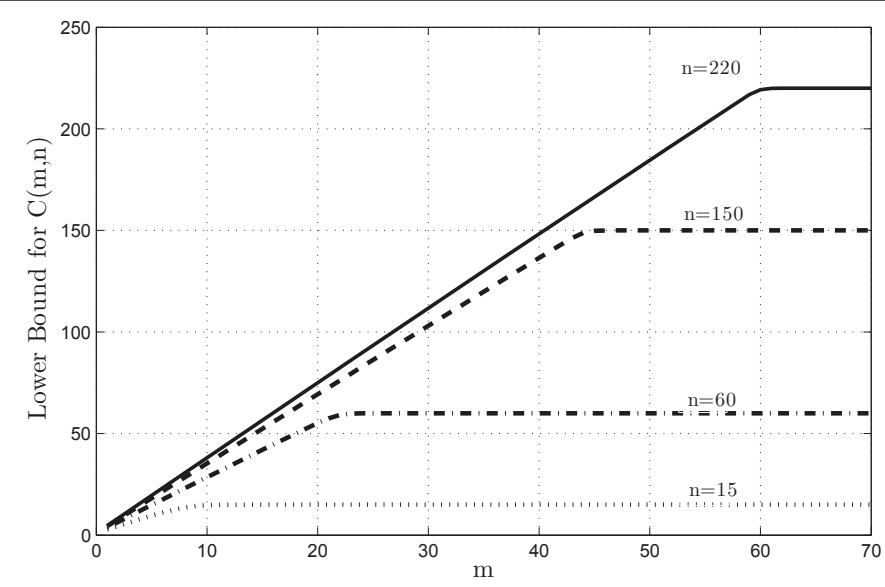

Figure 7 Plots of channel capacity lower bounds versus chip rate $m$ for various number of users $n$.

For the special case, when the additive noise is Gaussian, the above theorem can be stated in a more explicit way by setting $q(x)=\frac{\gamma}{2}\left|\frac{x}{\sigma}\right|^{2} \log e$ and the resulting lower bound is shown below:

$$
\begin{aligned}
C(m, n, \mathcal{I}, \mathcal{S}, \eta) \geq & \sup _{\pi, p} \sup _{\gamma}[-m(\gamma \log e \\
& -\log (1+\gamma))-\log \mathbb{E}_{\tilde{X}}\left(\left(\left.\mathbb{E}_{b}\left(\mathrm{e}^{\frac{-\gamma r^{2}}{2(1+\gamma) m}}\right) b^{T} \tilde{X}\right|^{2}\right)\right] .
\end{aligned}
$$

For special cases, where the user alphabets of the inputs and the arbitrary signature matrices are finite, a lower bound is presented in $[20,21]$. The same authors have also obtained this lower bound for any noise distribution.

For example, when our input vectors and signature matrix are binary (COW case), the following inequality presents the sum capacity lower bound for any arbitrary noise distribution $f$ and any arbitrary function $q$ :

$$
\begin{aligned}
C(m, n, f) \geq & n-m \mathbb{E}\left(q\left(N_{1}\right)\right) \\
& -\log \left(\sum_{k=0}^{n}\left(\begin{array}{l}
n \\
k
\end{array}\right)\left(\mathbb{E}\left(2^{-q\left(N_{1}-\frac{2 s_{k}}{\sqrt{m}}\right)}\right)\right)^{m}\right),
\end{aligned}
$$

where $s_{k}$ is the sum of $k$ independent random variables taking \pm 1 with equal probability.

In $[20,21]$, the authors considered the function $q(x)$ to be equal to $-\gamma \log (f(x))$ where $f$ is the pdf of additive Gaussian noise with variance $\sigma^{2}$. Now, suppose we denote the capacity in this case by $C_{G}\left(m, n, \sigma^{2}\right)$, then we have the following family of lower bounds

$$
\begin{aligned}
C\left(m, n, \sigma^{2}\right) \geq n-m \gamma \log (\sqrt{e}) \\
-\log \left(\sum_{k=0}^{n}\left(\begin{array}{l}
n \\
k
\end{array}\right)\left(\sum_{j=0}^{k} \frac{\left(\begin{array}{l}
k \\
j
\end{array}\right)}{2^{k}} \frac{e^{-2\left(\frac{2 j-k}{\sigma \sqrt{m}}\right)^{2}\left(\frac{\gamma}{1+\gamma}\right)}}{\sqrt{1+\gamma}}\right)^{m}\right) .
\end{aligned}
$$

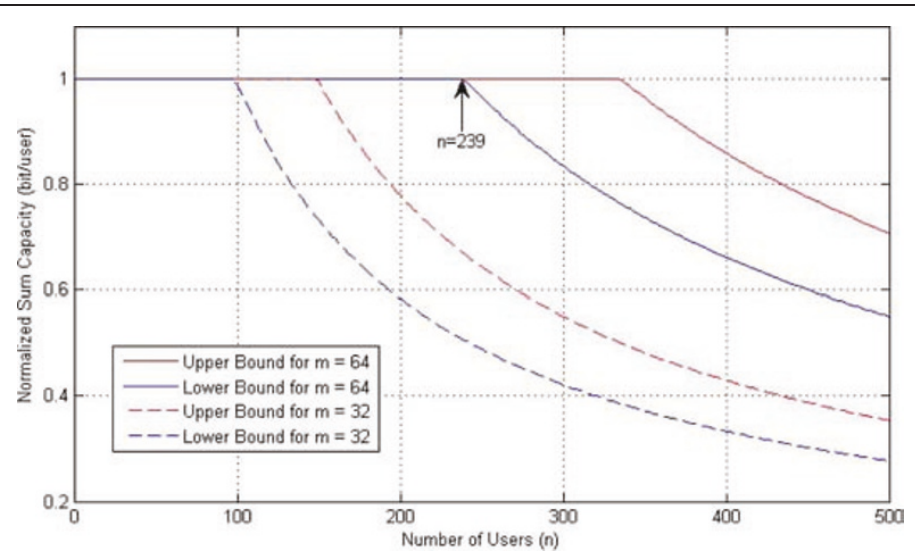

Figure 8 The lower and upper bounds for the normalized channel capacity versus the number of users $n$ for different spreading gain $m$ in a noiseless system with binary input. 


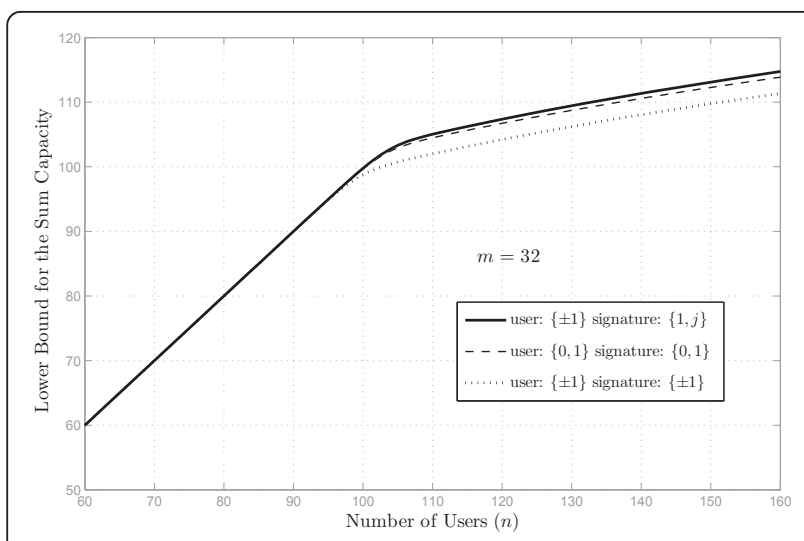

Figure 9 The sum capacity lower bound versus the number of users for binary input and binary signature matrices when all the probabilities are equal to $1 / 2$ for $m=32$.

\section{Conjectured upper bounds for the sum capacity of CDMA} systems for the noisy case

An upper bound is derived in $[20,21]$ for a general mode when the user inputs are finite in the presence of noise. However, we just review a conjectured upper bound for a special case where the input vectors and signature matrices are binary $(\mathrm{COW})$ that was introduced in [43].
The following theorem shows this conjectured upper bound:

Theorem 10 For any symmetric pdf function $f$, we have

$$
C(m, n, f) \leq \min (n, m(h(\tilde{f})-h(f)),
$$

where

$$
\tilde{f}(x)=\sum_{j=0}^{n} \frac{\left(\begin{array}{l}
n \\
j
\end{array}\right)}{2^{n}} f\left(x-\frac{2 j-n}{\sqrt{m}}\right),
$$

and $h(f)$ is the differential entropy of the distribution $f$. For the noiseless case, the usual entropy was used instead of the differential entropy.

For the special case when the noise has Gaussian distribution, $\tilde{f}$ becomes

$$
\tilde{f}(x)=\sum_{j=0}^{n} \frac{\left(\begin{array}{l}
n \\
j
\end{array}\right)}{2^{n}} e^{-\frac{\left(x-\frac{2 j-n}{\sqrt{m}}\right)^{2}}{2 \sigma^{2}}} .
$$

Then, we have:

$$
C_{G}\left(m, n, \sigma^{2}\right) \leq \min (n, m(h(\tilde{f})-\log (\sqrt{2 \pi e}))) .
$$

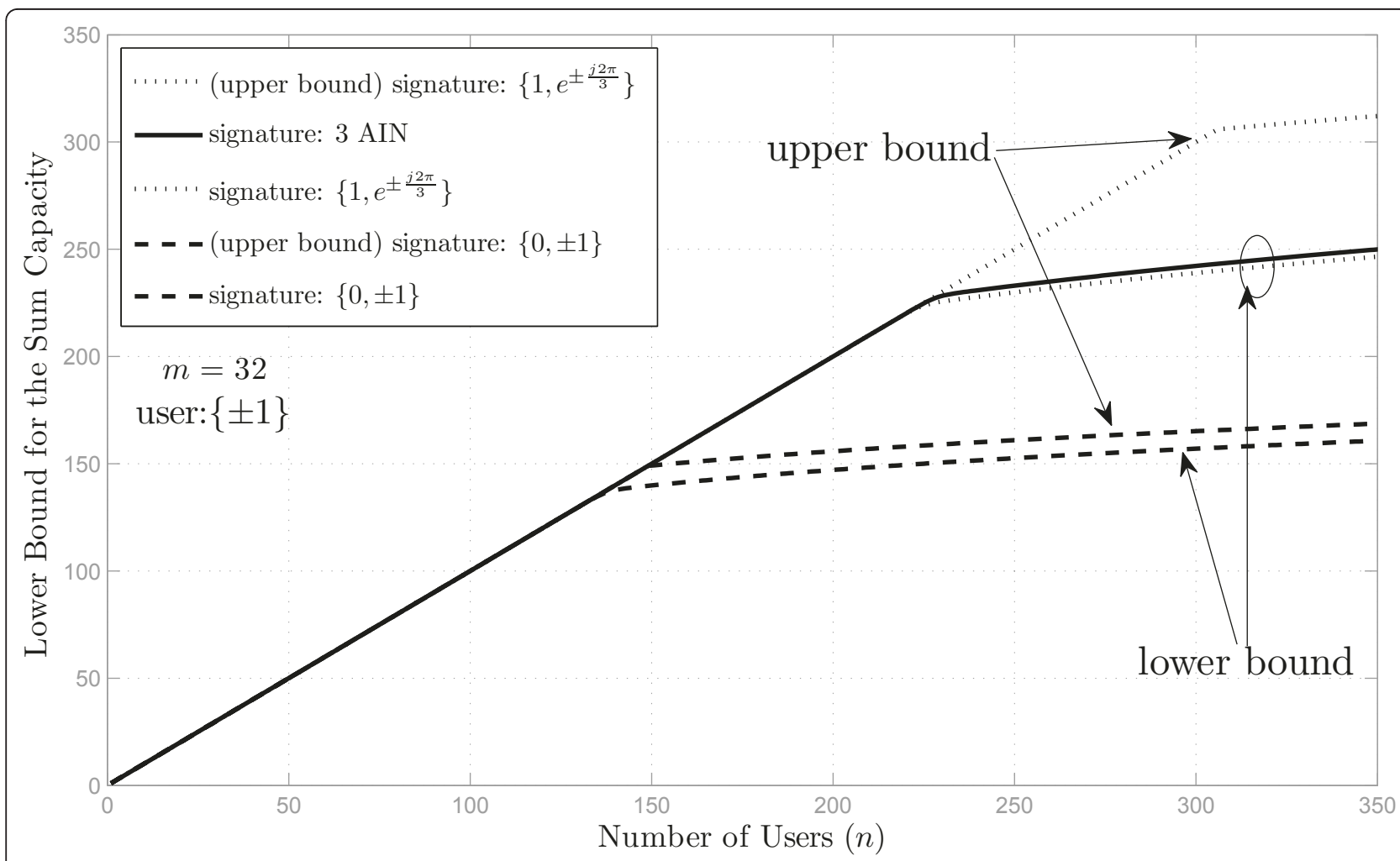

Figure 10 The sum capacity lower and upper bounds versus the number of users for binary input and ternary signature matrices when all the probabilities are equal. 


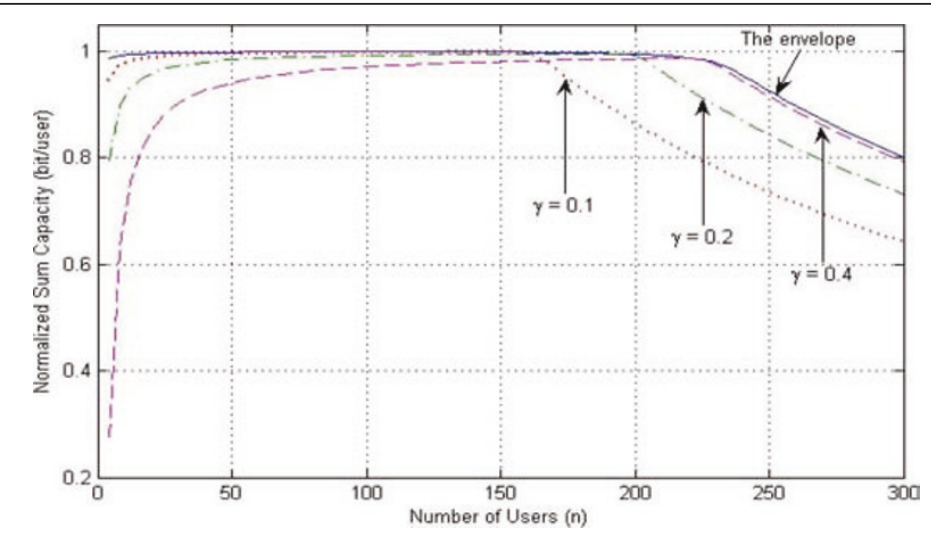

Figure 11 A family of lower bounds for the sum capacity of an AWGN channel using different $\gamma$ s and their envelope versus number of users $n$ when $E_{b} / N_{0}=8 \mathrm{~dB}$ and the spreading gain is 64 .

For some other noise distributions, the capacity upper bounds are derived in [21,43]. In Figure 11, the normalized sum channel capacity is shown for three values for $\gamma$. It can be concluded that for larger values of $\gamma$, the capacity of the channel increases.

As mentioned in the previous subsection, the channel capacity in the small scale system depends on the input and signature alphabets. In Figure 12, this dependence is shown in a noisy channel for binary input and binary signature alphabets.

\section{Asymptotic analysis of CDMA systems}

The asymptotic analysis of CDMA channels is referred to the case in which the number of users and the spreading factor tend to infinity while their ratio $(\beta)$ remains constant. The asymptotic case which is also called large scale system [39-42,57], is being studied by many recent works. The base of these works are related to replica theory derived from statistical physics $[39,58]$. In the replica method, a quantity called free energy is used which indicates the cumulative generating function carrying all the information about the statistics of the system and is defined as follows:

$$
\mathcal{F}_{m}(Y, \mathbf{C})=\frac{1}{m} \log _{e} Z(Y, \mathbf{C}),
$$

where

$$
\left.Z(Y, \mathbf{C})=\sum_{X} p(X) e^{\left(-\frac{1}{2 \sigma^{2}}\left\|Y-m^{-\frac{1}{2}} \mathbf{C X}\right\|^{2}\right.}\right) .
$$

This quantity has self averaging property. In communication systems, it means that in the asymptotic case, the differential entropy normalized by the users is equal to its average. Using this assumption, the capacity of the large system channel was derived as follows:

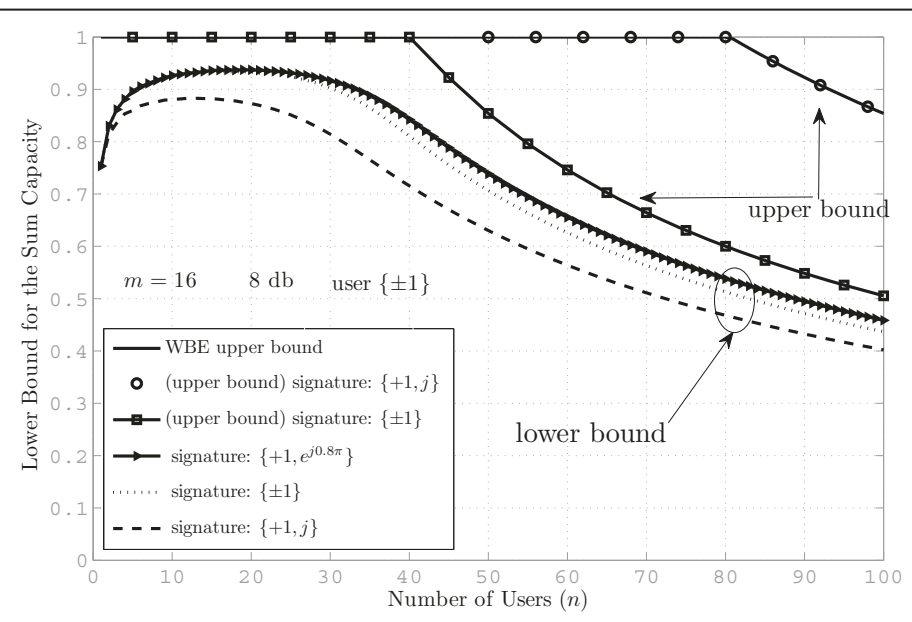

Figure 12 The sum capacity lower bound versus the number of users for binary input and binary signature matrix when all the probabilities are equal to $1 / 2$ for $m=16$ and $\eta=8 \mathrm{~dB}$. 


$$
C=\lim _{n \rightarrow \infty} \frac{1}{n} I\left(X_{1}, X_{2}, \ldots, X_{n} ; Y\right)=-\left(\mathcal{F}_{0}+\frac{1}{2 \beta}\right) .
$$

This expression can be applied for both binary and Gaussian distributed inputs. For the Gaussian input case, the expression is somewhat trivial but for the binary input case, the capacity is shown to be

$$
\lim _{m \rightarrow \infty} C=\min _{t \in[0,1]} \frac{\lambda}{2}(1+t)-\frac{1}{2 \beta} \lambda \sigma^{2}-\int \frac{e^{-\frac{z^{2}}{2}}}{\sqrt{2 \pi}} \ln (2 \cosh (\sqrt{\lambda} z+\lambda)) \mathrm{d} z,
$$

where

$$
\lambda=\frac{1}{\sigma^{2}+\beta(1-t)}
$$

and

$$
\left.t=\int \frac{\mathrm{e}^{-\frac{z^{2}}{2}}}{\sqrt{2 \pi}} \tanh (\sqrt{\lambda} z+\lambda)\right) \mathrm{d} z .
$$

This equation does not always yield a unique value for the capacity. This phenomenon is called phase coexistence and it occurs for $\beta$ values greater than 1.49. Tanaka considered the lowest solution to be the actual capacity. Montanari and Tse [40] used a new sparse signature scheme to prove the correctness of Tanaka's capacity for the binary input case for $\beta \leq 1.49$, where the above mentioned expression for $t$ has unique solution. Furthermore, they proved that for these values of $\beta$, optimal detection can be achieved using Belief Propagation (BP).

It was shown in [39] that as long as the channel capacity of the Gaussian input system is less than 1, it will be almost equal to the binary input system capacity for large $\beta$. But, since the binary input system capacity should not exceed 1 , the channel capacity saturates in this case to 1 , when the equivalent Gaussian input system capacity exceeds 1 .

The replica method is nonrigorous and the channel capacities obtained from this method are conjectured. In [41], it is proved that Tanaka's expression is an upper bound for the actual channel capacity for all values of $\beta$ using an interpolation method. The authors of [41] have also proved that the channel capacity for the large system limit $(\mathbf{C})$ concentrates to its mean $\left(\mathbb{E}_{S}\{\mathbf{C}\}\right)[42]$. In $[40,42]$ the authors have also proved that the sum channel capacity is independent of the signature alphabet for large scale systems.

In [24] decoding techniques were studied for the large scale system also using the replica method. For the MUD scheme, the authors have devised a technique to convert multi user detection into single user detection with some modified parameters for AWGN parameters as shown below:

$$
C_{\text {sep }}(\beta)=\beta \mathbb{E}\left\{I\left(\eta^{\prime} \mathrm{snr}\right)\right\},
$$

in which

$$
\square\left(\eta^{\prime} \mathrm{snr}\right)=\mathbb{D}\left(p_{Z \mid X, \mathrm{snr} ; \eta^{\prime}}|| p_{Z \mid \mathrm{snr} ; \eta^{\prime}} \mid p_{X}\right),
$$

where snr is the single user SNR, $\eta$ ' is the multiuser efficiency and $Z=\sqrt{\operatorname{snr}} X+\frac{N}{\sqrt{\eta^{\prime}}}$.

In the same paper, an expression for the channel capacity with optimal joint decoding (MUD) over separate decoding was derived in the large system limit as shown below:

$$
C_{\text {joint }}(\beta)=C_{\text {sep }}(\beta)+\left(\eta^{\prime}-1\right) \log e-\log \eta^{\prime} .
$$

Finally, it was concluded that for large scale systems, successive decoding with an individually optimal detection front end achieves the CDMA channel capacity with arbitrary inputs. For the special case of Gaussian inputs, the sum channel capacity can be achieved with Minimum Mean Square Error (MMSE) decoding.

Independently, the bounds for the asymptotic sum channel capacity were derived without using the replica method. The following inequality shows the lower bound for the asymptotic sum channel capacity that is derived in $[20,21]$ :

Let $b$ and $\tilde{X}$ be vectors of length $n$ with i.i.d. entries of distributions $\pi(\cdot)$ and $\tilde{p}(\cdot)$, respectively. Then

$$
\begin{aligned}
& \lim _{\substack{n, m \rightarrow \infty \\
n, m \rightarrow \beta}} \frac{1}{n}\left[-m \gamma \log e-\log \mathbb{E}_{\bar{x}}\left(\left(\mathbb{E}_{b}\left(\frac{e^{\frac{-\gamma^{2}}{2(1+\gamma) m^{b} b^{2} x^{2}}}}{1+\gamma}\right)\right)^{m}\right)\right] \\
& =\operatorname{supl}_{\gamma} \inf _{\hat{p}(1), \mu_{x} \mu_{\hat{p}}=0}\left\{\mathbb{D}(\hat{p} \mid \tilde{p})-\frac{1}{\beta}(\gamma \log e-\log (1+\gamma))\right. \\
& \left.\left.+\frac{1}{2 \beta}\left(\log \left(1+\frac{2 \beta \eta \gamma \lambda_{1}}{(1+\gamma) \sigma_{p}^{2}\left(\sigma_{\pi}^{2}+\mu_{\pi}^{2}\right)}\right)+\log \left(1+\frac{2 \beta \eta \gamma \lambda_{2}}{(1+\gamma) \sigma_{p}^{2}\left(\sigma_{\pi}^{2}+\mu_{\pi}^{2}\right)}\right)\right)\right\}\right\},
\end{aligned}
$$

where $\hat{p}$ the empirical distribution of $\tilde{p}$, and $\lambda_{1}, \lambda_{2}$ are eigenvalues of the covariance matrix of a random variable which has the distribution of the product of two independent variables with distribution $\hat{p}$ and $\pi$, and $\mathbb{D}(\cdot \| \cdot)$ is the Kullback-Leibler distance. The term in the limit is the sum capacity lower bound for finite alphabets which is derived in [21].

In [21], the authors have also obtained an upper bound for the sum channel capacity for the binary input case in the presence of additive noise with arbitrary distribution. The following inequality shows this upper bound

$$
\lim _{\substack{n / m=\beta \\ n, m \rightarrow \infty}} c(m, n, f) \leq \min \left\{1, \frac{1}{\beta}\left(h\left(N_{1}+\sqrt{\beta} Z\right)-h\left(N_{1}\right)\right)\right\},
$$

where $Z$ is a Gaussian random variable independent of $N_{1}$. If the additive noise is Gaussian, then $N_{1}$ is a Gaussian random variable with variance $\sigma^{2}$. 
Thus, $h\left(N_{1}\right)=\frac{1}{2} \log \left(2 \pi e \sigma^{2}\right)$

$h\left(N_{1}+\sqrt{\beta} Z\right)=\frac{1}{2} \log \left(2 \pi e\left(\sigma^{2}+\beta\right)\right)$. Hence,

$$
\lim _{\substack{n / m=\beta \\ n, m \rightarrow \infty}} c(m, n, f) \leq \min \left\{1, \frac{1}{2 \beta} \log \left(1+\frac{\beta}{\sigma^{2}}\right)\right\} .
$$

The above upper bound is reminiscent of the Shanon capacity for an AWGN channel where $\frac{1}{\beta}=\frac{m}{n}$ is the normalized bandwidth and the SNR $=\frac{\beta}{\sigma^{2}}$. As $\beta$ approaches zero, the above bound goes to $\log \frac{e}{2 \sigma^{2}}$. This bound is appropriate for low SNR (for $\frac{E_{b}}{N_{0}} \leq 1.593 \mathrm{~dB}$, this upper bound will be less than 1 bit per user). However, for $\beta \leq$ 1 , the actual channel capacity reaches the single user capacity.

For the binary input case, the normalized sum capacity bounds are plotted in Figure 13. Tanaka's result lies between the conjectured lower and upper bounds introduced in [43]. As $\beta$ increases, Tanaka's capacity tends to reach the upper bound and the lower and upper bounds become tighter.

Figure 14 shows the asymptotic lower bound for the normalized sum capacity versus $\eta$ for QPSK inputs and for $\beta=1$ and 3. As $\beta$ increases, the lower bound that was introduced in [21] becomes closer to Guo-Verdu's result ( $\eta$ is defined in (18)).

For a noiseless channel, the sum capacity was derived in [21] where the authors compared the results of Tanakas's asymptotic capacity which was shown in $[24,39-42,59]$, with their bounds. The asymptotic sum channel capacity for a fixed $\beta$ is equal to [22]

$$
\lim _{\substack{n / m=\beta \\ n, m \rightarrow \infty}} \frac{1}{n} c(m, n)=1
$$

As it was shown in the first section, as $m$ becomes large, the rate of increase of $n$ is much faster than $m$ for COW codes. Thus, the assumption of reaching the full capacity in (50) is justifiable. For nonbinary inputs and signature matrices, the asymptotic lower capacity bound can be shown from the following theorem [21]

\section{Theorem 11}

$$
\begin{gathered}
\lim _{\substack{n /(m \log n) \rightarrow \zeta \\
n, m \rightarrow \infty}} \frac{1}{n} C(m, n, \mathcal{I}, \mathcal{S}) \geq \min _{\mathcal{J} \subseteq \tilde{I}} \\
\left\{\frac{\operatorname{rank}(\mathcal{J} \cdot S)}{2 \zeta}-\log \tilde{p}(\mathcal{J})\right\},
\end{gathered}
$$

where $\mathcal{J} \cdot \mathcal{S}=\{j s \mid j \in \mathcal{J}, s \in \mathcal{S}\}$ and $\tilde{p}(\mathcal{J})=\sum_{j \in \mathcal{J}} \tilde{p}(j)$ and for a set of numbers $\Lambda, \operatorname{rank}(\Lambda)$ denotes the dimension of $\Lambda$ as a set of vectors over the field of rational numbers $\mathcal{Q}$.

For the special case when $\mathcal{I}=\mathcal{S}=\{ \pm 1\}$ and $\pi$ and $p$ are uniform distributions on $\mathcal{I}$ and $\mathcal{S}$ (binary case), we have $\tilde{\mathcal{I}}=\{-2,0,2\}$ and $\tilde{p}(-2)=\tilde{p}(2)=\frac{1}{4}$ and $\tilde{p}(0)=\frac{1}{2}$. Thus, the above bound is simplified as shown below:

$$
\lim _{\substack{n /(m \log n) \rightarrow \zeta \\ n, m \rightarrow \infty}} \frac{1}{n} C(m, n) \geq \min \left\{1, \frac{1}{2 \zeta}\right\}
$$

In [43], an upper bound was also derived and the result is given below:

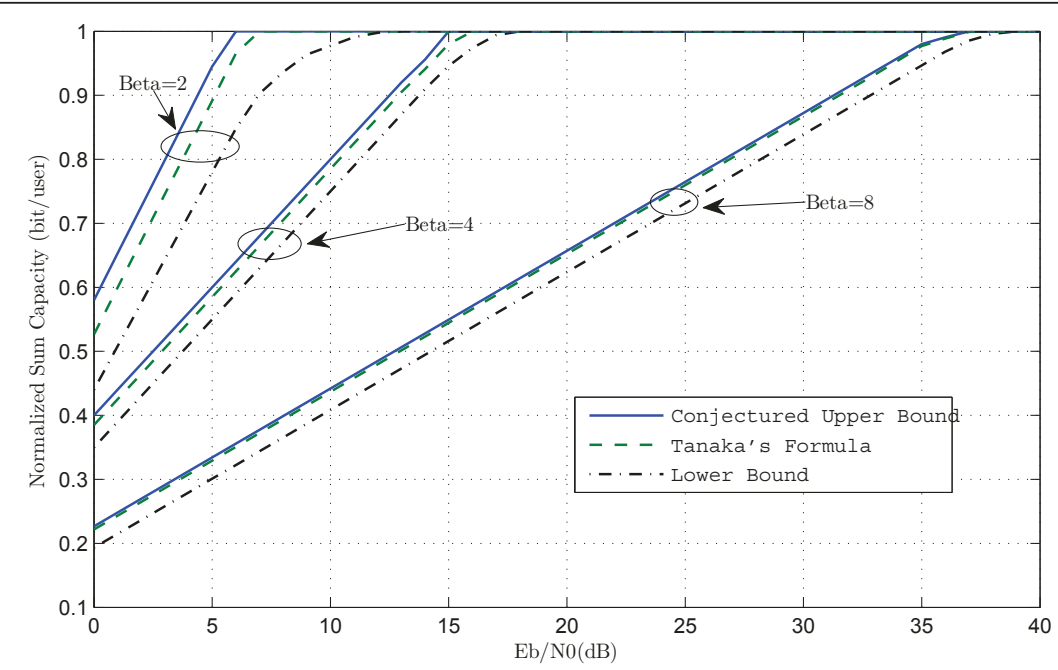

Figure 13 The normalized sum capacity bounds versus $E_{b} / N_{0}$ in the limit when $n$ and $m$ go to infinity for binary input and $\beta=2,4$ and 8. Depending on the values of $\beta$, Tanaka's bound is somewhere between other bounds but closer to the conjectured upper bound as $\beta$ increases. As $\beta$ increases, the lower and upper bound and Tanaka's bound become very tight. 


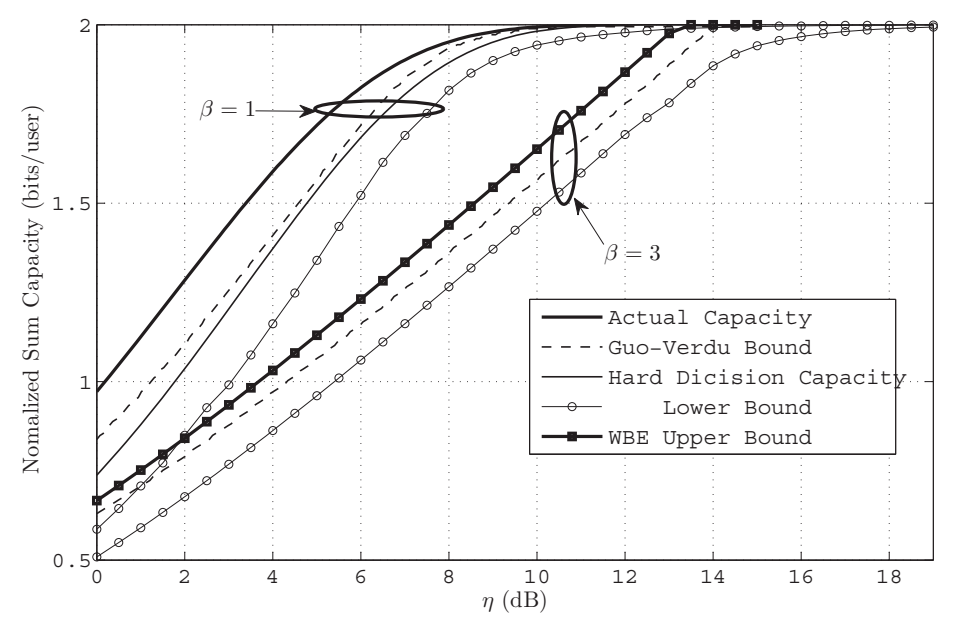

Figure 14 The asymptotic lower bounds for the normalized sum capacity versus signal to noise ratio for QPSK inputs for $\beta=1$ and 3 In this figure, our lower bound is compared to that of Guo-Verdu and the single user sum capacity.

$$
\lim _{\substack{n /(m \log n)=\zeta \\ n, m \rightarrow \infty}} \frac{1}{n} C(m, n) \leq \min \left\{1, \frac{1}{2 \zeta}\right\}
$$

The above results for binary matrices show that the lower and upper capacity bounds approach each other asymptotically, and therefore, we have the actual capacity. stop

In Figure 15, the normalized sum channel capacity for small to medium scale systems is shown. This figure shows that small to medium scaled systems cannot be accurately estimated by the asymptotic lower bound for high values of $\zeta$.

\section{Overloaded CDMA systems with near- far effects}

So far, we have reviewed the cases in which we have perfect power control. This means that the received power of each user is equal at the receiver. But in reality this is not true since the distance between the base station and the users vary from user to user. Due to fading problems in the transmission channel, the user powers are not equal. In this section, we will first consider a class of COW codes that are resistant to near-far effects. Then, we will discuss the channel capacity bounds for such systems. At the end, we will survey optimum power estimation methods in systems with near-far effects for highly overloaded CDMA systems.

A Errorless codes for CDMA systems with near- far effects In the previous sections, the channel model is considered as $Y=\mathbf{C} X+N$, where $\mathbf{C}$ is the signature matrix. Without perfect power control, we cannot assume the input vectors to be \pm 1 at the receiver anymore. By considering near-far effects, the channel is modeled as follows:

$$
Y=\mathrm{CP}^{1 / 2} X+N,
$$

where $\mathbf{P}^{1 / 2}=\operatorname{diag}\left(p_{1}^{1 / 2}, p_{2}^{1 / 2}, \ldots, p_{n}^{1 / 2}\right)$ is the matrix of the received amplitude of each user. All nonzero entries of $\mathbf{P}^{\mathbf{1} / \mathbf{2}}$ belong to the interval $[1-\delta, 1+\delta]$, where $0 \leq \delta$ $\leq 1$. In [48], the authors have presented an additive channel model instead of (54) as shown below:

$$
Y=\mathbf{C}(X+Z)+N \text {, }
$$

where $Z$ is an $n \times 1$ random vector with entries in the interval $[-\delta, \delta]$. In order to have errorless transmission in the absence of noise, a signature matrix should be found such that the intersection of the transformation of the sets $X_{1}+[-\delta, \delta]^{n}$ and $X_{2}+[-\delta, \delta]^{n}$ are empty for different $X_{1}$ 's and $X_{2}$ 's.

The following two definitions are needed for performance comparison [48].

Definition 1 For a CDMA system with near-far effects, we define the Power Control Factor (PCF) as 10 $\log \frac{\mathbb{E}(g)^{2}}{\operatorname{var}(g)}$, where $g$ is the general pdf of the entries of $\mathbf{P}^{1 /}$ 2 . This concept shows the amount of power fluctuations of the users. In the special case where we assume the entries of $\mathbf{P}^{1 / 2}$ to be uniformly distributed in the interval $[1-\delta, 1+\delta]$, we obtain:

$$
\mathrm{PCF}_{\mathrm{dB}}=10 \log \frac{3}{\delta^{2}} \text {. }
$$

Definition $2 \delta_{\text {sup }}$ is defined as $\delta_{\text {sup }}(\mathbf{C})=\sup \{\delta \mid \mathbf{C} \cdot(X$ $\left.+[-\delta, \delta]^{n}\right)$ are disjoint for all $\left.X \in\{ \pm 1\}^{n}\right\}$. Consequently,

$$
\mathrm{PCF}_{\text {inf }}=10 \log \frac{3}{\delta_{\text {sup }}^{2}}
$$




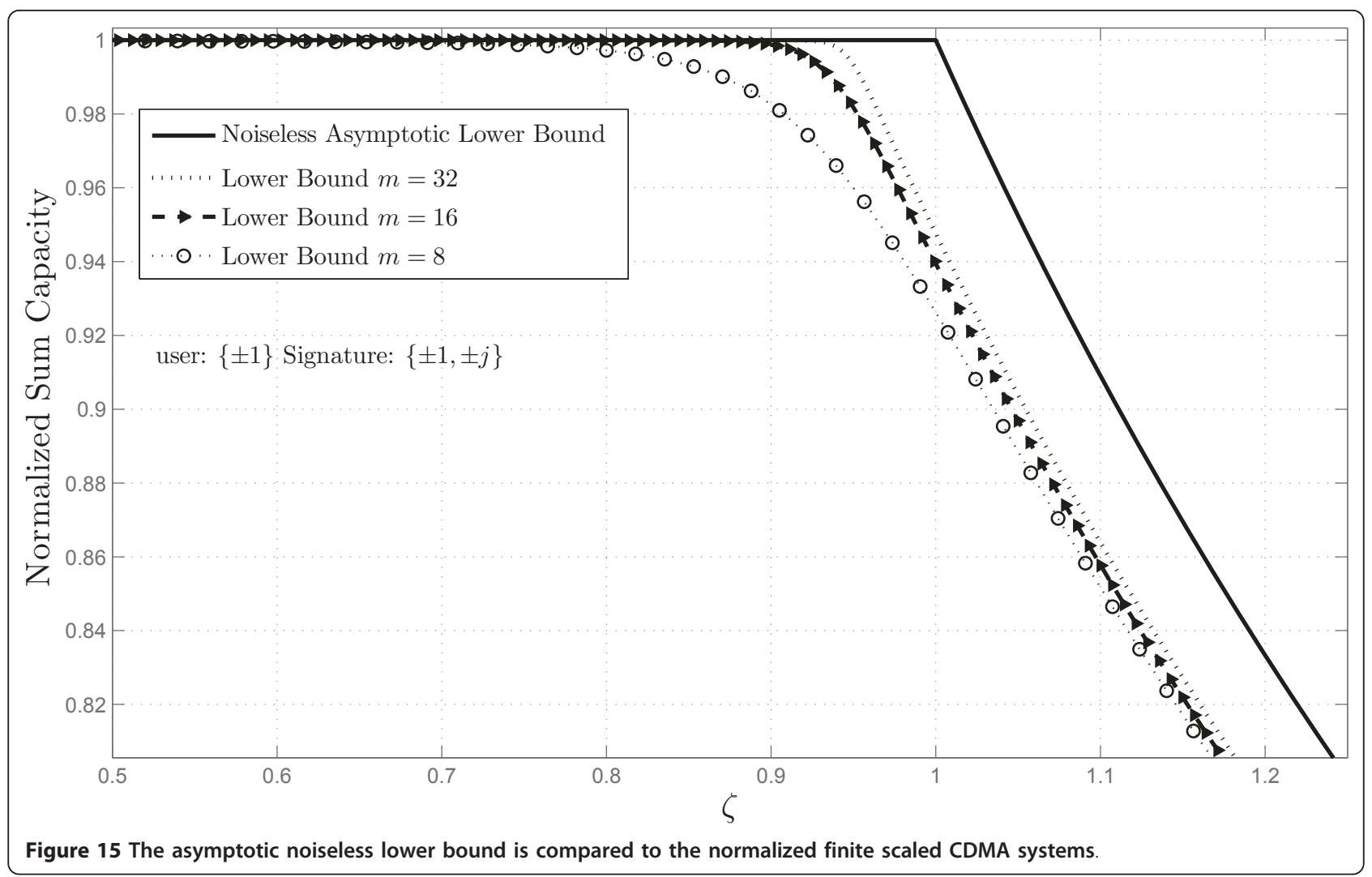

In [48] upper and lower bounds for $\delta_{\text {sup }}(\mathbf{C})$ is found

$$
\begin{aligned}
& \delta_{\text {sup }}\left(\mathbf{C}_{m \times n}\right) \leq \frac{1}{2^{\frac{n}{m}}-1} \\
& \delta_{\text {sup }}(\mathbf{C}) \geq \frac{\min _{X \in S}\|\mathbf{C} X\|}{\max _{X \in\{ \pm 1\}^{n}}\|\mathbf{C X}\|}
\end{aligned}
$$

The above inequality shows that errorless transmission is possible for any $\delta$ such that

$$
\delta<\sup \left(\frac{\min _{X \in S}\|\mathbf{C X}\|}{\max _{X \in\{ \pm 1\}^{n}}\|\mathbf{C} X\|}\right)
$$

For the $\mathbf{C}_{8 \times 13}$ COW matrix in Section II, the upper and lower bounds have been evaluated. The upper bound is 0.48 and the lower bound is 0.13 and the simulation results shows that $\delta_{\text {sup }}$ is 0.23 [48].

By using the lower bound, small sized near-far resistant signature matrices can be found that guarantee errorless transmission for a noiseless channel. But the evaluation of bounds on $\delta_{\text {sup }}$ for large sized signature matrices with this method requires a high amount of computation. It is shown in [48] that the $\delta_{\text {sup }}$ of a large signature matrix created with the method of Theorem 2 from a smaller one, is equal to the original matrix.

$$
\delta_{\text {sup }}\left(\mathbf{P} \otimes \mathbf{C}_{m \times n}\right)=\delta_{\text {sup }}(\mathbf{C})
$$

Since $\delta_{\text {sup }}$ is equal for both signature matrices, a new lower bound can be found by substituting the new matrix in (59). Thus, we have

$$
\delta_{\text {sup }}(\mathbf{C}) \geq \frac{\min _{X \in\{0, \pm 1\}^{k n}-\{0\}^{k n}}\|\mathbf{P} \otimes \mathbf{C X}\|}{\max _{X \in\{ \pm 1\}^{k n}} \| \mathbf{P} \otimes \mathbf{C X \|}},
$$

where $\mathbf{P}$ is a $k \times k$ invertible matrix.

The ML decoding of this kind of codes is very similar to the Tensor Decoding Algorithm. In this case, the received vector is $Y=\mathbf{C}(X+Z)+N$. Each entry of $Z$ takes a random value belonging to the interval $[-\delta, \delta]$. In order to consider the worst case, uniform distribution for $Z$ is assumed; the time samples of $Z$ are also assumed to be independent. This assumption is much worse than what occurs in practice because in practical situations, user powers are constant at least for a short period of time. Thus, the time samples of $Z$ should be correlated. By using the generalized Central Limit Theorem, $W=\mathbf{C Z}+N$ is approximated with a Gaussian vector with zero mean and auto covariance matrix $\frac{\delta^{2}}{3} \mathbf{C C}^{T}+\sigma^{2} \mathbf{I}$. Thus, the channel modeled as $Y=\mathbf{C} X+$ 
$W$. Similar to the previous sections, the Tensor Decoding Algorithm can be used to decode the received vector.

The performance of robust codes against near-far effects is shown in Figure 16 for the binary case. It can be concluded from this figure that as PCF increases, the performance of such codes improves.

\section{B Channel capacity bounds for CDMA systems with near- far effects}

In the previous subsection, we reviewed codes that guarantee errorless transmission through a noisy channel. In [24], the sum channel capacity with near-far effects was derived for Gaussian inputs and nonbinary signature alphabets. In [49], an asymptotic lower bound and a conjectured upper bound were derived for the binary case. It was assumed that the diagonal matrix $\mathbf{P}^{1 / 2}$ has Gaussian distribution with mean equal to 1 . Referring to the channel model shown in (55), the entries of $Z$ are independent with Gaussian distribution and zero mean. For underloaded codes $(\beta<1), C(\beta, \sigma, \rho)$ (where $\sigma^{2}$ and $\rho^{2}$ are the variance of $N$ and $Z$, respectively) is known, while for the overloaded case $(\beta>1)$, the determination of $C(\beta, \sigma, \rho)$ is an open problem. Similar to previous sections, a lower bound and a conjectured upper bound are reviewed for the channel capacity. In order to derive a new upper bound, the lower bound derived in [49] is as follows:

$$
\begin{gathered}
C(\beta, \sigma, \rho) \geq 1-\inf _{\gamma} \sup _{t \in[0,1]}\left[H(t)+\frac{1}{2 \beta}(\gamma \log e\right. \\
\left.\left.-\log \left(1+\gamma\left(1+\frac{4 t \beta}{\theta^{2}}\right)\right)\right)\right]
\end{gathered}
$$

where $\theta^{2}=(\sqrt{\beta}+1)^{2} \rho^{2}+\sigma^{2}$.

Since, for a given variance, the Gaussian distribution has the maximum entropy, the above lower bound is

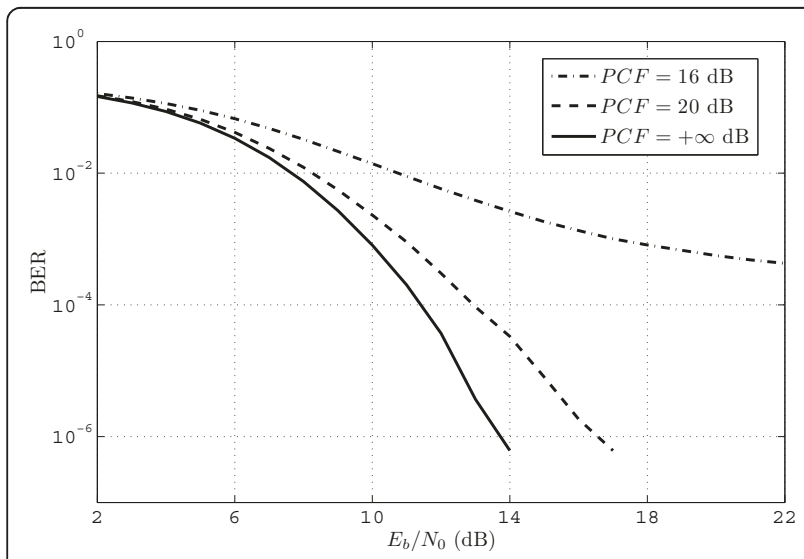

Figure 16 BER versus $E_{b} / N_{0}$ for binary CDMA system with 64 chips and 104 users (binary signature/binary inputs). the lower bound for the sum capacity of any system with near-far variance equal to $\rho^{2}$.

The following inequality shows the conjectured upper bound for the sum channel capacity in the presence of near-far effects

$$
C(\beta, \sigma, \rho)=\min \left\{h(\hat{\gamma})-h(\gamma), \frac{1}{2 \beta} \log \left(1+\frac{\beta}{w^{2}}\right)\right\}
$$

where $w^{2}=(\sqrt{\beta}-1)^{2} \rho^{2}+\sigma^{2}$.

In Figure 17, the lower bounds for an overloaded binary CDMA system with $\beta=2$ and several values of PCF are presented. This figure shows the effect of PCF on the channel capacity. As it can be predicted, greater PCF results in improved capacity.

Figure 18 shows a comparison of the proposed bounds including Tanaka's bound for $\beta=4$. This figure shows that as $E_{b} / N_{0}$ increases, Tanaka's capacity reaches the upper bound and the upper and lower bounds become tighter.

\section{Power estimation}

In the previous subsection, we have surveyed a class of codes for which we can handle the near-far effects without actually estimating the received power of each user at the receiver. In this subsection, we consider a power estimating method which is suitable for overloaded CDMA systems [50]. The aim of this method is to estimate the power matrix $\mathbf{P}$ in (54). In order to achieve this goal, power estimation based on ML is employed.

In [50], the authors have proved that if $\overline{\bar{S}}$ is an $\frac{m(m+1)}{2} \times n$ matrix whose rows are the entry-by-entry multiplication of the rows of the signature matrix $\mathbf{S}$, the estimated power matrix is unique if and only if

$$
\operatorname{rank}(\overline{\overline{\mathrm{S}}})=n \text {. }
$$

Since the rank of a matrix cannot exceed the number of its columns, the maximum number of users for

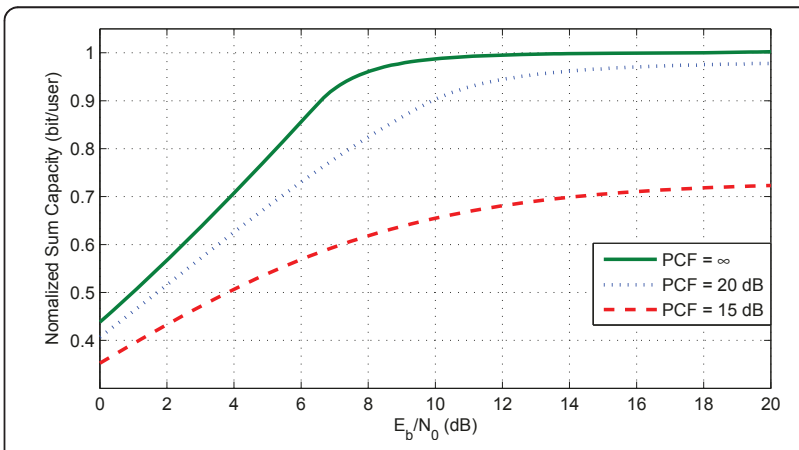

Figure 17 The normalized sum capacity sum capacity lower bounds versus $E_{b} / N_{0}$ for $\beta=2$ and various values of PCF. 


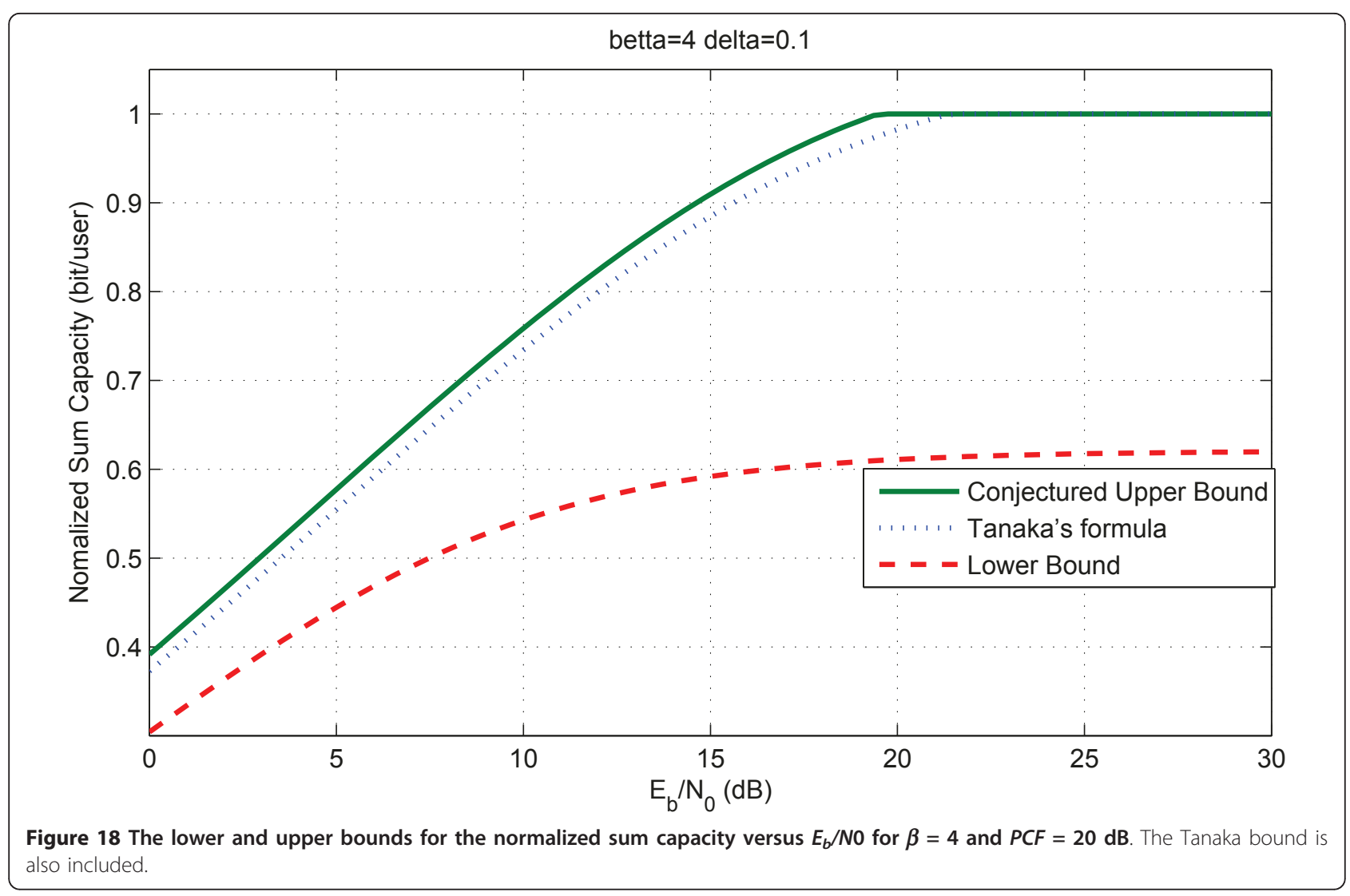

optimum power estimation is equal to $\frac{m \times(m+1)}{2}$.

Although this method is optimum for obtaining ML power estimation, its computational complexity is very high. A suboptimum method which has a lower complexity is also shown in [50]. If the channel noise is assumed to be white, this method can be employed for systems with unknown noise covariance matrix. In this case, the maximum number of users should not exceed $\frac{m(m-1)}{2}$ in order to have the desired power estimation.

In [50], the power change of a user with time, due to physical movement or channel characteristic variation, has also been considered. This helps the estimating systems to track sudden power changes more rapidly. Moreover, the existence of suitable signature matrices has also been studied. It can be shown that this power estimation method is optimum only when the input alphabet is Gaussian. For the binary input case, an iterative estimating method is used in [50]. In each iteration, the actual power is obtained from the previous iteration before decoding the data of each user; the next iteration is performed from this data.

The performance of the suboptimum power estimation is shown in Figure 19; this figure shows that the performance of the estimation becomes better as the number of received vectors increases.

\section{Summary, conclusion, and future works A Summary}

In this tutorial review, an extensive survey of synchronous CDMA systems is provided and key issues related to such systems are discussed. In this subsection, a general summary of the previous sections is given:

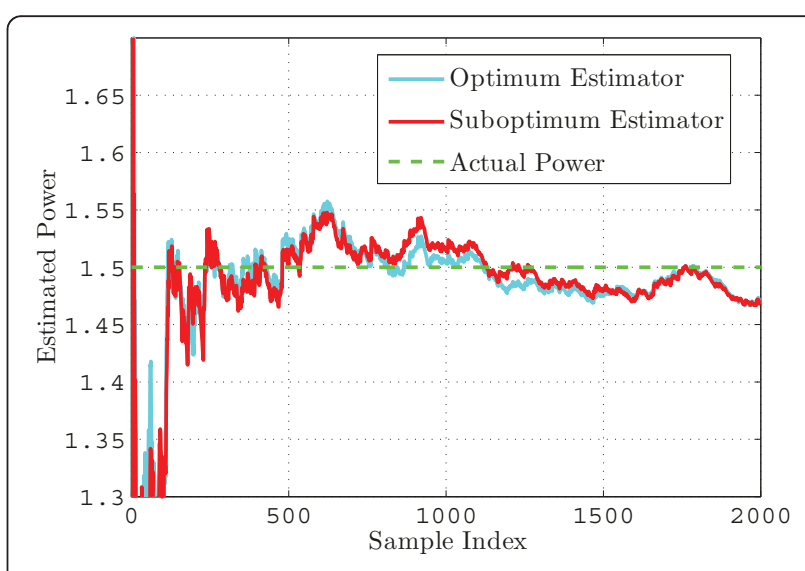

Figure 19 The $M L$ and suboptimum estimation of the power versus the number of received vectors $L$ in a system with $n=$ 12 and $m=8$ that uses a binary WBE signature matrix. 
In Section II, we discussed overloaded binary and nonbinary signature codes that provide errorless transmission in noiseless channels. The extension of these errorless codes to active user detection system is also discussed. An upper bound for the maximum number of users for a given chip rate is also derived in this section. An example of an errorless code of size $64 \times 193$ (for $m=64$ and $n=193$ ) was also given. But in the same section, it was concluded that for the chip rate $m$ equal to 64, more than 239 users cannot be supported for errorless transmission.

Section III is a survey of the sum channel capacity bounds for binary and nonbinary user inputs and signature matrices. The same section also reviews the bounds for the noiseless and noisy channels as well as the asymptotic cases.

Section IV is a review of errorless codes and sum capacity with near-far effects. This section discusses the same issues such as errorless transmission codes and channel capacity with near-far effects. To improve the robustness of $\mathrm{COW} / \mathrm{COO}$ codes against near-far effects and improve the channel capacity, channel power estimation is required. Since power control is an important power estimation for near-far effects, a subsection is devoted to power estimation methods. This section also derives the channel capacity for input Gaussian and nonbinary matrices as well as lower and upper bounds for binary inputs and binary matrices.

\section{B Conclusions}

CDMA has gained broad application in recent multiple access communication systems. The growth of demand and limited bandwidth has raised new issues in providing sufficient service to the growing number of subscribers. Overloaded CDMA is a proper solution for this problem. In overloaded CDMA, a larger number of users can be accommodated with the same signature size. This means that we can handle more users than TDMA and FDMA for the same bandwidth. COW/ COO codes are an important class of signature codes which can provide errorless transmission in noiseless channels. It can be shown that these codes have better performance than random and BWBE codes for the binary case. Also, The $\mathrm{COW} / \mathrm{COO}$ codes are resistant to near-far effects and each $\mathrm{COW} / \mathrm{COO}$ code has its own robustness.

Channel capacity analysis is also an important issue in recent works related to CDMA. For small scale systems, the sum channel capacity depends on the signature alphabets while for large scale systems, the channel capacity tends to be independent of the signature alphabets. Tanaka's sum capacity for binary and Guo-Verdu's sum capacity for nonbinary cases lie between lower and upper bounds in the asymptotic case. However, as the overloading factor $\beta$ increases, the sum capacity of Tanaka and Guo-Verdu approach the upper bound and the lower and upper bounds become tighter. Another important conclusion is that for small to medium scale systems, asymptotic values of Tanaka and Guo-Verdu cannot be used for the estimation of the sum channel capacity, and therefore, we have to use the lower and upper bounds introduced.

It can also be shown that that power estimation and control are possible for very highly overloaded systems.

\section{Future works}

Research topics that can be considered for future works are as follows: Although, Section II implies that ML decoding using Tensor Decoding Algorithm can be used for certain overloaded errorless codes, it is desirable to extend the overloading factor beyond the ones discussed in this paper. The same problem can be also extended to active user detection case. Despite the development of lower and upper bounds for the sum channel capacity in Section III, it is desirable to find the actual sum capacity for any given number of users, chip rates, and signal-to-noise ratios. In addition, it is very desirable to find the actual capacity regions. In active user detection systems, channel capacity should be explored with and without side information about the active users. The capacity bounds and the capacity region should be also extended to the near-far effects as discussed in Section IV. In the near-far effects, channel capacity should be derived considering channel state information. Finally, the asynchronous case should be explored for all the above cases. Generalized user vectors [60] is another interesting topic to be studied.

\section{Endnotes}

Endnote a. In general, by wireless binary CDMA, we mean the signature codes and the input data are binary $\{ \pm 1\}$; while for optical CDMA systems, the binary elements are $\{0,1\}$.

Endnote $\mathbf{b}$. If the number of users is less than the signature length, the system is called underloaded. If the number of users is more than the signature length, it is called overloaded and the case where they are equal is called fully loaded.

Endnote c. Systems with the ability to detect users that are inactive in the current transmission are called systems with active user detection. Inactive users are modeled as users that transmit zero.

Endnote d. In Equation 1, $\otimes$ represents the Kronecker product.

Competing interests

The authors declare that they have no competing interests. 
Received: 13 December 2010 Accepted: 16 August 2011 Published: 16 August 2011

\section{References}

1. T Ojanpera, R Prasad, Wideband CDMA For Third Generation Mobile Communications: Universal Personal Communications (Artech House, Inc., Norwood, 1998)

2. F Chung, J Salehi, V Wei, Optical orthogonal codes: design, analysis and applications. IEEE Trans Inf Theory, 35(3), 595-604 (1989). doi:10.1109/ 18.30982

3. J Salehi, Code division multiple-access techniques in optical fiber networks. I. Fundamental principles. IEEE Trans Commun. 37(8), 824-833 (1989). doi:10.1109/26.31181

4. J Salehi, Emerging OCDMA communication systems and data networks [Invited]. J Opt Netw. 6(9), 1138-1178 (2007). doi:10.1364/JON.6.001138

5. S Verdu, Multiuser Detection (Cambridge University Press, Cambridge, 1998)

6. A Kapur, M Varanasi, Multiuser detection for overloaded CDMA systems. IEEE Trans Inf Theory 49(7), 1728-1742 (2003). doi:10.1109/TIT.2003.813562

7. S Moshavi, Multi-user detection for DS-CDMA communications. IEEE Commun Mag. 34(10), 124-136 (1996). doi:10.1109/35.544334

8. F Chung, J Salehi, V Wei, Optical orthogonal codes: design, analysis and applications. IEEE Trans Inf Theory 35(3), 595-604 (1989). doi:10.1109/ 18.30982

9. S Verdú, S Shamai, Spectral efficiency of CDMA with random spreading IEEE Trans Inf Theory 45(2), 622-640 (1999). doi:10.1109/18.749007

10. A Grant, P Alexander, Random sequence multisets for synchronous codedivision multiple-access channels. IEEE Trans Inf Theory 44(7), 2832-2836 (1998). doi:10.1109/18.737515

11. P Pad, M Faraji, F Marvasti, Constructing and decoding GWBE codes using Kronecker products. IEEE Commun Lett. 14(1), 1-3 (2010)

12. G Karystinos, D Pados, in Proceedings of the IEEE Global Telecommunication Conference (GLOBECOM). Minimum Total-Squared-Correlation Design of DSCDMA Binary Signature Sets, vol. 2. (San Antonio, Texas, USA, 2001), pp. 801-805

13. G Karystinos, D Pados, New bounds on the total squared correlation and optimum design of DS-CDMA binary signature sets. IEEE Trans Commun. 51(1), 48-51 (2003). doi:10.1109/TCOMM.2002.807628

14. $\mathrm{L}$ Welch, Lower bounds on the maximum cross correlation of signals (Corresp). IEEE Trans Inf Theory 20(3), 397-399 (1974). doi:10.1109/ TIT.1974.1055219

15. H Sari, F Vanhaverbeke, M Moeneclaey, Extending the capacity of multiple access channels. IEEE Commun Mag. 38(1), 74-82 (2000). doi:10.1109/ 35.815455

16. F Vanhaverbeke, M Moeneclaey, H Sari, DS/CDMA with two sets of orthogonal spreading sequences and iterative detection. IEEE Commun Lett. 4(9), 289-291 (2000). doi:10.1109/4234.873489

17. H Sari, F Vanhaverbeke, M Moeneclaey, Multiple access using two sets of orthogonal signal waveforms. IEEE Commun Lett. 4(1), 4-6 (2000). doi:10.1109/4234.823531

18. F Vanhaverbeke, M Moeneclaey, H Sari, in Proceedings of the IEEE International Conference on Communications (ICC). Increasing CDMA Capacity Using Multiple Orthogonal Spreading Sequence Sets and Successive Interference Cancellation, vol. 3. (New York, NY, USA, 2002), pp. 1516-1520

19. H Sari, F Vanhaverbeke, M Moeneclaey, Extending the capacity of multiple access channels. IEEE Commun Mag. 38(1), 74-82 (2000). doi:10.1109/ 35.815455

20. K Alishahi, S Dashmiz, P Pad, F Marvasti, Design of overloaded cdma and the derivation of sum capacity bounds for synchronous cdma systems. Conditionally accepted for publication in the IEEE Transactions on Information Theory (2011)

21. K Alishahi, S Dashmiz, P Pad, F Marvasti, M Shafinia, M Mansouri, The Enigma of CDMA Revisited. Arxiv preprint arXiv:1005.0677 (2010)

22. P Pad, F Marvasti, K Alishahi, S Akbari, A class of errorless codes for overloaded synchronous wireless and optical CDMA systems. IEEE Trans Inf Theory 55(6), 2705-2715 (2009)

23. P Pad, M Soltanolkotabi, S Hadikhanlou, A Enayati, F Marvasti, in Proceedings of the IEEE International Conference on Communications (ICC). Errorless Codes for Over-Loaded CDMA with Active User Detection (Dresden, Germany, 2009), pp. 1-6
24. D Guo, S Verdu, Randomly spread CDMA: asymptotics via statistical physics. IEEE Trans Inf Theory 51(6), 1983-2010 (2005). doi:10.1109/TIT.2005.847700

25. R Ahlswede, in Proceedings of the Second International Symposium on Information Theory, ed. by Petrov BN (Multi-way communication channels. (Tsahkadsor, Armenia, USSR: Akadémiai Kiadó, Budapest, 1971), pp. 23-51

26. R Ahlswede, The capacity region of a channel with two senders and two receivers. The Ann Probab. 2(5), 805-814 (1974). doi:10.1214/aop/ 1176996549

27. H Liao, Multiple Access Channels (Hawaii University, Honolulu, 1972)

28. T Cover, Some advances in broadcast channels. Adv Commun Syst. 4, 229-260 (1975)

29. T Cover, J Thomas, J Wiley, Elements of Information Theory, vol. 1 (Wiley Online Library, London, 1991)

30. A Wyner, Recent results in the Shannon theory. IEEE Trans Inf Theory 20(1), 2-10 (1974). doi:10.1109/TIT.1974.1055171

31. S Verdu, in Proceedings of the 24th Allerton Conference. Capacity region of Gaussian CDMA channels: the symbol-synchronous case (1986), pp. 1025-1034

32. S Verdu, The capacity region of the symbol-asynchronous Gaussian multiple-access channel. IEEE Trans Inf Theory 35(4), 733-751 (1989). doi:10.1109/18.32152

33. S Verdú, S Shamai, Spectral efficiency of CDMA with random spreading. IEEE Trans Inf Theory 45(2), 622-640 (1999). doi:10.1109/18.749007

34. M Rupf, J Massey, Optimum sequence multisets for synchronous codedivision multiple-access channels. IEEE Trans Inf Theory 40(4), 1261-1266 (1994). doi:10.1109/18.335940

35. P Viswanath, $\vee$ Anantharam, Optimal sequences and sum capacity of synchronous CDMA systems. IEEE Trans Inf Theory 45(6), 1984-1991 (1999). doi:10.1109/18.782121

36. S Ponnaluri, T Guess, Effects of spreading and training on capacity in overloaded CDMA. IEEE Trans Commun. 56(4), 523-526 (2008)

37. M Medard, The effect upon channel capacity in wireless communications of perfect and imperfect knowledge of the channel. IEEE Trans Inf Theory 46(3), 933-946 (2000). doi:10.1109/18.841172

38. B Hassibi, B Hochwald, How much training is needed in multiple-antenna wireless links? IEEE Trans Inf Theory 49(4), 951-963 (2003). doi:10.1109/ TIT.2003.809594

39. T Tanaka, A statistical-mechanics approach to large-system analysis of CDMA multiuser detectors. IEEE Trans Inf Theory 48(11), 2888-2910 (2002). doi:10.1109/TIT.2002.804053

40. A Montanari, D Tse, in Proceedings of the Information Theory Workshop ITW IEEE. Analysis of Belief Propagation for Non-Linear Problems: The Example of CDMA (or: How to prove Tanaka's formula). (Punta del Este, 2006), pp. $160-164$

41. S Korada, N Macris, Tight bounds on the capacity of binary input random CDMA systems. IEEE Trans Inf Theory 56(11), 5590-5613 (2010)

42. S Korada, $\mathrm{N}$ Macris, in Proceedings of the IEEE International Symposium on Information Theory (ISIT). On the Concentration of the Capacity for a Code Division Multiple Access System (Nice, France, 2007), pp. 2801-2805

43. K Alishahi, F Marvasti, V Aref, P Pad, Bounds on the sum capacity of synchronous binary CDMA channels. IEEE Trans Inf Theory 55(8), 3577-3593 (2009)

44. Y Cho, J Lee, Analysis of an adaptive SIC for near-far resistant DS-CDMA IEEE Trans Commun. 46(11), 1429-1432 (1998). doi:10.1109/26.729384

45. U Madhow, Blind adaptive interference suppression for the near-far resistant acquisition and demodulation of direct-sequence CDMA signals. IEEE Trans Signal Process. 45(1), 124-136 (1997). doi:10.1109/78.552211

46. F Zheng, S Barton, Near-far resistant detection of CDMA signals via isolation bit insertion. IEEE Trans Commun. 43(234)، 1313-1317 (1995)

47. U Madhow, M Honig, On the average near-far resistance for MMSE detection of direct sequence CDMA signals with random spreading. IEEE Trans Inf Theory. 45(6), 2039-2045 (1999). doi:10.1109/18.782129

48. M Shafinia, P Kabir, P Pad, S Mansouri, F Marvasti, in Proceedings of the IEEE International Conference on Communications (ICC)2. Errorless Codes for CDMA Systems with Near-Far Effect. (Cape Town, South Africa, 2010), pp. $1-5$

49. P Pad, M Shafinia, S Mansouri, P Kabir, F Marvasti, Bounds for the Sum Capacity of Binary CDMA Systems in Presence of Near-Far Effect. Arxiv preprint arXiv:1003.5345 (2010) 
50. O Mashayekhi, D Nashtaali, P Pad, S Moghaddasi, F Marvasti, New Power Estimation Methods for Highly Overloaded Synchronous CDMA Systems. Arxiv preprint arXiv:1104.4612v1 (2011)

51. S Dashmiz, M Takapoui, P Pad, F Marvasti, in Proceedings of the IEEE International Symposium on Information Theory (ISIT). New Bounds for the Sum Capacity of Binary and Nonbinary Synchronous CDMA Systems (Austin, Texas, USA, 2010), pp. 2093-2097

52. M Rupf, J Massey, Optimum sequence multisets for synchronous codedivision multiple-access channels. IEEE Trans Inf Theory. 40(4), 1261-1266 (1994). doi:10.1109/18.335940

53. J Tropp, I Dhillon, R Heath Jr, Finite-step algorithms for constructing optimal CDMA signature sequences. IEEE Trans Inf Theory 50(11), 2916-2921 (2004). doi:10.1109/TT.2004.836698

54. Z Xu, Blind identification of co-existing synchronous and asynchronous users for CDMA systems. IEEE Signal Process Lett. 8(7), 212-214 (2001). doi:10.1109/97.928681

55. P Pad, A Mousavi, A Goli, F Marvasti, Simplified MAP-MUD for Active User CDMA. IEEE Commun Lett. 15(99), 599-601 (2011)

56. R Learned, A Willsky, D Boroson, Low complexity optimal joint detection for oversaturated multiple access communications. IEEE Trans Signal Process. 45(1), 113-123 (1997). doi:10.1109/78.552210

57. S Korada, N Macris, in Proceedings of the Allerton Conference on Communications, Control, and Computing. On the Capacity of a Code Division Multiple Access System (Monticello, USA, 2008)

58. H Nishimori, Statistical Physics of Spin Glasses and Information Processing: an Introduction (Oxford University Press, USA, 2001)

59. T Tanaka, in Proceedings of the IEEE International Symposium on Information Theory (ISIT). Replica Analysis of Performance Loss Due to Separation of Detection and Decoding in CDMA Channels (Seattle, Washington, USA, 2006), pp. 2368-2372

60. W Mow, Recursive constructions of detecting matrices for multiuser coding: A unifying approach. IEEE Trans Inf Theory 55(1), 93-98 (2009)

doi:10.1186/1687-1499-2011-62

Cite this article as: Hosseini et al:: A review on synchronous CDMA systems: optimum overloaded codes, channel capacity, and power control. EURASIP Journal on Wireless Communications and Networking 2011 2011:62.

\section{Submit your manuscript to a SpringerOpen ${ }^{\circ}$ journal and benefit from:}

- Convenient online submission

- Rigorous peer review

- Immediate publication on acceptance

- Open access: articles freely available online

- High visibility within the field

- Retaining the copyright to your article

Submit your next manuscript at $\gg$ springeropen.com 